\title{
Two Theorems on Extensions of Holomorphic Mappings
}

\author{
Phillip A. Griffiths (Princeton)
}

\section{Introduction and Table of Contents}

In this paper we shall prove two theorems about extending holomorphic mappings between complex manifolds. Both results involve extending such mappings across pseudo-concave boundaries. The first is a removable singularities statement for meromorphic mappings into compact Kähler manifolds. The precise result and several illustrative examples are given in Section 1. The second theorem is a Hartogs'-type result for holomorphic mappings into a complex manifold which has a complete Hermitian metric with non-positive holomorphic sectional curvatures. This theorem answers one of Chern's problems posed at the Nice Congress [3]. The precise statement and further discussion is given in Section 4.

The proofs of both theorems use the class of pluri-sub-harmonic (p.s.h.) functions, which is intrinsically defined on any complex manifold [9]. The second proof is rather elementary and essentially relates the p.s.h. functions on the domain of $f$ to the curvature assumption on the image manifold. The first theorem is technically a little more delicate and makes use of the removable singularity theorems for analytic sets due to Bishop-Stoll [14] together with the strong estimates available for the amount of singularity which the Levi form of a p.s.h. function may have at an isolated singularity of such a function.

At the end of this paper there are two appendices. The first contains a brief survey of some removable singularity theorems for holomorphic mappings between complex manifolds. In the second appendix we give an informal discussion of the general problem of defining the "order of growth" of a holomorphic mapping and using this notion to study such maps. The basic open question here is what might be termed "Bezout's theorem for holomorphic functions of several variables," and this problem is discussed and precisely formulated there.

It is my pleasure to acknowledge many helpful discussions with $\mathrm{H}$. Wu concerning the material presented below. In particular, several of the ideas and results in Appendix 2 were communicated to me by him. 


\section{Contents}

1. Statement and Discussion of Theorem I . . . . . . . . . . . . 28

2. Preliminary Results for the Proof of Theorem I . . . . . . . . . . . . 30

3. Proof of Theorem I . . . . . . . . . . . . . . . . . . . . . . . . 34

4. Statement and Discussion of Theorem II . . . . . . . . . . . . . . . . 38

5. Proof of Theorem II . . . . . . . . . . . . . . . . . . . . . 41

Appendix I. Survey of Some Removable Singularity Theorems . . . . . . . . . 48

Appendix II. Some Remarks on the Order of Growth of Holomorphic Mappings . . 50

References . . . . . . . . . . . . . . . . . . 62

\section{Statement and Discussion of Theorem I}

Let $M$ and $N$ be connected complex manifolds of complex dimensions $m$ and $n$ respectively and where $M$ is assumed to be compact. We recall that a meromorphic mapping

$$
f: N \rightarrow M
$$

is given by an irreducible analytic subset (the graph of $f$ )

$$
\Gamma \subset N \times M
$$

together with a proper analytic subset $S \subset N$ and a holomorphic mapping

$$
f: N-S \rightarrow M
$$

such that $\Gamma$ restricted to $(N-S) \times M$ is exactly the graph of $f$. Thus $\pi_{N}: \Gamma \rightarrow N$ is a proper modification and $\pi_{M}: \Gamma \rightarrow M$ is a holomorphic mapping. Conversely, a holomorphic mapping $f$, which as in (1.2) is defined on the complement of a proper subvariety $S \subset N$, will be said to be meromorphic if the closure $\vec{\Gamma}_{f}$ in $N \times M$ of the graph $\Gamma_{f} \subset(N-S) \times M$ is an analytic subvariety of $N \times M$. In this case, we may also say that $S$ is a removable singularity for $f$ as a meromorphic mapping from $N$ to $M$. We are primarily interested in removing singularities for holomorphic mappings (1.2) when $\operatorname{codim}(S) \geqq 2$. Here are a few simple examples to illustrate the problem.

Example 1. If $M=\mathbf{C}$ or $\mathbf{P}_{1}$, then if $\operatorname{codim}(S) \geqq 2$ it is always possible to remove the singularities of a holomorphic mapping $f: N-S \rightarrow M$. In this case, $f$ is just a holomorphic or meromorphic function respectively, and the result is classical (cf. Narasimhan [11], p. 133).

Example 2. If $M$ is a projective algebraic variety and $f$ a holomorphic mapping as in (1.2), then it is easily seen that $S$ is a removable singularity for $f$ if, and only if, the pull-backs $f^{*}(\varphi)$ of all rational functions on $M$ extend to meromorphic functions on $N$. Thus, if $\operatorname{codim}(S) \geqq 2$, then $S$ is a removable singularity by Example 1 above.

Example 3. If $M=\mathbf{C}^{m} /$ (lattice) is a complex torus, then any holomorphic mapping $(1.2)$ with $\operatorname{codim}(S) \geqq 2$ extends to a holomorphic 
mapping $f: N \rightarrow M$. This follows from: (a) the fact that, given $x \in S$, there is a neighborhood $U$ of $x$ in $N$ such that the fundamental group $\pi_{1}(U-U \cap S)=0$; (b) the monodromy theorem; and (c) Example 1 above.

Example 4. We recall that the Hopf manifold $M$ is obtained from factoring $\mathbf{C}^{\boldsymbol{m}}-\{0\}$ by the properly-discontinuous infinite cyclic group generated by the linear transformation

$$
\left(z_{1}, \ldots, z_{m}\right) \rightarrow\left(2 z_{1}, \ldots, 2 z_{m}\right) .
$$

If we take $N=\mathbf{C}^{m}$ and $S=\{0\}$, then the quotient mapping

$$
\mathrm{C}^{m}-\{0\} \stackrel{f}{\longrightarrow} \mathrm{M}
$$

does not extend meromorphically across the origm. I his follows from: (a) the fact that every annular ring

$$
\frac{1}{2^{k}} \leqq\|z\|<\frac{1}{2^{k-1}} \quad(k=1,2, \ldots)
$$

maps onto $M$, and (b) the observation that, if $\operatorname{dim} N=\operatorname{dim} M$ and if $f: N-S \rightarrow M$ extends to a meromorphic mapping of $N$ into $M$, then

$$
\operatorname{dim}[f(x)] \leqq m-1
$$

for all $x \in S$. Here we have used the notation

$$
f(x)=\operatorname{proj}_{M}\left(\bar{\Gamma}_{f} \cdot\{x\} \times M\right)
$$

for the compact analytic subvariety of $M$ into which $x$ is mapped by $f$.

From this example we see that, even though the problem of removing the singularities of $f$ in (1.2) is in some sense a local question in $N \times M$, it is false for $\operatorname{codim}(S) \geqq 2$ without making global assumptions on $M$. Our main result is

Theorem I. Let $B_{n}^{*}=\left\{z \in \mathbf{C}^{n}: 0<\|z\| \leqq 1\right\}$ be the punctured ball in $\mathbf{C}^{n}$, and $f: B_{n}^{*} \rightarrow M$ a holomorphic mapping into a compact Kähler manifold $M^{1}$. Then $f$ extends to a meromorphic mapping from the ball $B_{n}=$ $\{z:\|z\| \leqq 1\}$ into $M$, provided that $n \geqq 3$.

Remarks. There are two criticisms of this result, which we should like to discuss here. The first is regarding the restriction $n \geqq 3$ instead of $n \geqq 2$ as one would have hoped for. This condition arose because of the vanishing Theorem (2.6) below, which does not hold for $n=2$. It has recently been proved by Shiffman that the necessary cohomology class (but not the whole group) vanishes for our problem when $n=2$, so that Theorem I is also true in this case. Shiffman's result is discussed following (2.6).

${ }^{1}$ To say that $f: B_{n}^{*} \rightarrow M$ is holomorphic means, by definition, that $f$ is holomorphic in the open region $0<\|z\|<1+\varepsilon$ for some $\varepsilon>0$. 
The second criticism, which is more serious, is that we should have an extension theorem for any holomorphic mapping $f: N-S \rightarrow M$ whenever $M$ is compact Kähler and $\operatorname{codim}(S) \geqq 2$. However, our proof only works in case $S$ is 0 -dimensional, and the author has been unable to decide if the more general result is true (cf. Problem 0 at the end of $\S 3$ below).

It is perhaps worth remarking that there are two definitions of meromorphic mappings. The one we have given above is due to Remmert (Math. Ann. 133, $367(1957)$ ). The other definition, due to Stoll (Math. Z. 67, $468(1955)$ ), is that $f: N-S \rightarrow M$ is meromorphic if, for every analytic curve $C \subset N$ such that $C \cap S$ has dimension zero, if follows that $f: C-C \cap S \rightarrow M$ extends holomorphically to $C$. For algebraic varieties $M$, these definitions coincide, but I am not sure of the general relationship. At any event, our proof of Theorem I will show that $f: N-S \rightarrow M$ $(\operatorname{codim}(S) \geqq 3, M$ compact Kähler) extends meromorphically in the sense of Stoll.

A final remark is concerning the reason for proving a result, such as Theorem I, for Kähler manifolds when certainly the most interesting examples of such are the algebraic varieties where the theorem is well known and proved by standard methods. Of course, this is a personal matter, but for me the point is that usually proving a result using the Kählerian condition forces one to localize much more than is necessary in algebraic geometry, and this frequently leads to a more interesting proof and new insight. Hopefully this is somewhat the case here.

\section{Preliminary Results for the Proof of Theorem I}

a) On the Theorem of Bishop-Stoll

Let $V$ be a complex manifold, $W \subset V$ an analytic subvariety, and $X$ a pure $k$-dimensional analytic subvariety in $V^{*}=V-W$.

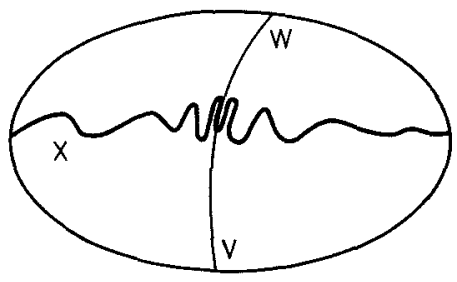

Fig. 1

The question we wish to discuss is: When is $\bar{X}$ (closure in $V$ ) an analytic subvariety of $V$ ? One very nice answer is provided by the theorem of Bishop-Stoll [14], which goes as follows. Let $d s_{V}^{2}=\sum_{i, j} h_{i j}(v) d v_{i} d \bar{v}_{j}$ 
be an Hermitian metric on $V$ and $\omega=\frac{\sqrt{-1}}{2}\left(\sum_{i, j} h_{i j}(v) d v_{i} \wedge d \bar{v}_{j}\right)$ the corresponding $(1,1)$ form. We will say that $X$ has locally finite area in $V$ if, given $x \in W$ there is a neighborhood $U$ of $x$ in $V$ such that, letting $U^{*}=U \cap V^{*}$,

$$
\int_{X \cap U^{*}} \omega^{k}<\infty
$$

(This condition is independent of the particular $d s_{V}^{2}$ on $\left.V.\right)^{2}$

(2.2) Theorem (Bishop-Stoll). The closure $\bar{X}$ is an analytic subvariety of $V$ if, and only if, $X$ has locally finite area in $V$.

Remark. We will discuss this result in the language of currents [9], and show how this leads fairly easily to a proof of (2.2) in the special case when $\operatorname{codim}(Z)=1(Z=X$ in the statement of (2.2)). Unfortunately, this will not cover the applications we have in mind. There is a general discussion of these matters in the paper "The currents defined by analytic varieties" by James King which will appear in Acta Mathematica.

Denote by $C^{q, q}\left(V^{*}\right)$ (respectively $\left.C^{q, q}(V)\right)$ the currents of type $(q, q)$ on $V^{*}$ (respectively on $V$ ). Letting $q=\operatorname{dim}\left(V^{*}\right)-k$ be the codimension of $Z$ in $V^{*}$, we see that $Z$ defines a current $T_{Z}^{*} \in C^{q, q}\left(V^{*}\right)$ by the formula

$$
T_{Z}^{*}\left(\alpha^{*}\right)=\int_{Z} \alpha^{*}
$$

where $\alpha^{*}$ is a $C^{\infty}$ form with compact support in $V^{*}$. The current $T_{Z}^{*}$ satisfies the equations

$$
d T_{Z}^{*}=0=d^{c} T_{Z}^{*}
$$

(Recall that $d^{c}=\sqrt{-1}(\bar{\partial}-\partial)$ so that $d d^{c}=2 \sqrt{-1} \partial \bar{\partial}$.)

Now it is easy to see that the condition that $Z$ have locally finite area in $V$ is exactly the condition that $T_{Z}^{*}$ extend to a current $T_{Z} \in C^{q, q}(V)$ defined by

$$
T_{Z}(\alpha)=\lim _{n \rightarrow \infty} T_{Z}^{*}\left(\alpha_{n}\right),
$$

where $\alpha$ is a $C^{\infty}$ form with compact support in $V$ and where the $\alpha_{n}$ are compactly supported $C^{\infty}$ forms in $V^{*}$ such that $\lim _{n \rightarrow \infty} \alpha_{n}=\alpha$ uniformly on $V$. Furthermore, it may be seen that

$$
d T_{Z}=0=d^{c} T_{Z}
$$

${ }^{2}$ For questions about integration over analytic varieties we refer to [14]. Observe that the integral in (2.1) is (essentially) the Hausdorff $2 k$-volume of $X$ relative to the given metric on $V$. 
as currents on $V$. (This is less trivial and in particular requires that the Hausdorff $(2 k-1)$-measure of $\bar{Z} \cap W$ be zero; cf. [14].)

Suppose now that $\operatorname{codim}(Z)=1$ so that $T_{Z} \in C^{1,1}(V)$ is a current satisfying (2.3). Taking $V$ to be a polycylinder in $\mathbf{C}^{n}$, which is permissible since the problem is local in $V$, we may find a current $\theta \in C^{0,0}(V)$ which satisfies the equation of currents

$$
d d^{c} \theta=T_{Z} .
$$

It follows that $\theta$ is a pluri-sub-harmonic (abbreviated p.s.h.) function in $V^{*}$ which extends as a current, and therefore as a p. s. h. function, to $V^{3}$. If we let $\omega=\partial \theta$, then $d \omega=0$ and an easy argument shows that we may define a holomorphic function $f(z)$ on $V$ by the formula

$$
f(z)=\exp \left(\int_{z_{0}}^{z} \omega\right) .
$$

The equation $f=0$ defines the closure $\bar{Z}$ of $Z$ in $V$, and therefore proves (2.2) in this special case.

Unfortunately, the interplay between p.s.h. functions and subvarieties of higher codimension is quite non-linear and so the above argument does not seem to readily generalize.

For our applications, we shall need the following corollary of (2.2):

(2.4) Proposition. Let $M$ be a compact, complex manifold with Hermitian metric $d s_{M}^{2}$ and associated $(1,1)$ form $\omega$. Let $f: B_{n}^{*} \rightarrow M$ be a holomorphic mapping of the punctured ball into $M$ and set $\omega_{f}=f^{*}(\omega)$. Then $f$ extends to a meromorphic mapping of $B_{n}$ into $M$ if, and only if, we have the estimate

$$
\int_{B_{n}^{*}} \omega_{f}^{k} \wedge \varphi^{n-k}<\infty \quad(k=1, \ldots, n)
$$

where $\varphi=\frac{\sqrt{-1}}{2}\left(\sum_{j=1}^{n} d z_{j} \wedge d \bar{z}_{j}\right)$ is the usual Euclidean $(1,1)$ form on $\mathbf{C}^{n}$.

Proof. In Theorem (2.2) we take $V=B_{n} \times M, W=\{0\} \times M, X=\Gamma_{f}$ the graph of $f$, and on $V$ the product metric whose associated $(1,1)$ form is $\varphi+\omega$. Then we have

$$
\int_{\Gamma_{f}}(\varphi+\omega)^{n}=\sum_{k=0}^{n}\left(\begin{array}{l}
n \\
k
\end{array}\right) \int_{B_{n}^{*}} \omega_{f}^{k} \varphi^{n-k}
$$

and the result follows by comparing this with (2.1) and (2.5).

Remark. Let us examine the term

$$
\int_{B_{n}^{*}} \omega_{f}^{n}
$$

${ }^{3}$ We shall recall the definition and elementary properties of p.s.h. functions in a little while. 
for the holomorphic mapping $f: \mathbf{C}^{n}-\{0\} \rightarrow M$ where $M$ is the Hopf manifold constructed in Example 4 of Section 1. From the definition, it is clear that

$$
\int_{\frac{1}{2^{k}} \leqq\|z\| \leqq \frac{1}{2^{k-1}}} \omega_{f}^{n}=\operatorname{vol}(M) .
$$

Thus we have the asymptotic formula

$$
\int_{\varepsilon \leqq\|z\| \leqq 1} \omega_{f}^{n} \sim \frac{1}{\varepsilon}(\log 2) \operatorname{vol}(M),
$$

which becomes infinite as $\varepsilon \rightarrow 0$.

\section{b) On the Cohomology of the Punctured Ball}

The result we shall need is this:

(2.6) Proposition. The cohomology groups $H^{1}\left(B_{n}^{*}, \mathcal{O}\right)=0$ for $n \neq 2$. Thus, for $n \neq 2$, if $\gamma$ is a $C^{\infty}(0,1)$ form on $B_{n}^{*}$ which satisfies $\bar{\partial} \gamma=0$, then there is a $C^{\infty}$ function $\eta$ on $B_{n}^{*}$ such that $\bar{\partial} \eta=\gamma$.

Remarks. The general result is the vanishing theorem

$$
H^{q}\left(B_{n}^{*}, \mathcal{O}\right)=0 \quad(q \neq 0, n-1),
$$

while the remaining group $H^{n-1}\left(B_{n}^{*}, \mathcal{O}\right)$ turns out to be infinite-dimensional. The vanishing statement (2.7) is proved in the paper of G. Scheja, Math. Ann. 144, 357 (1967).

Observe that (2.7) implies that $H^{1}\left(B_{n}^{*}, \mathcal{O}^{*}\right)=0$ for $n \geqq 3$; i.e. all holomorphic line bundles on $B_{n}^{*}$ are trivial for $n \geqq 3$. It is certainly not true that all line bundles are trivial on $B_{2}^{*}$; however, Shiffman has recently shown that any such line bundle $\mathbf{L}$ is trivial provided that it is positive in a suitable sense. In particular, he has proved that a $C^{\infty}(0,1)$ form $\gamma$ on $B_{2}^{*}$ which satisfies $\bar{\partial} \gamma=0, \partial \gamma \geqq 0$, is of the form $\gamma=\bar{\partial} \eta$ for some $C^{\infty}$ function on $B_{2}^{*}$. It is this result which leads to Theorem I in case $n=2$.

\section{c) Removable Singularities of Pluri-Sub-Harmonic Functions}

We recall a standard definition [9].

Definition. A function $\psi$ on a connected complex manifold $N$ is pluri-sub-harmonic (p.s.h.) if (i) $-\infty \leqq \psi<+\infty$ and $\psi \neq-\infty$; (ii) $\psi$ is upper-semi-continuous, and (iii) the restriction of $\psi$ to every holomorphic disc $\Delta \subset N$ is sub-harmonic on $\Delta^{4}$.

4 An equivalent definition is that (i) $\psi \in L_{\text {loc }}^{1}(N)$ should be a locally $L^{1}$ function on $N$; and (ii) if we consider such a $\psi$ as a current in $C^{0}(N)$, then we have

in the sense of currents.

$d d^{c} \psi \geqq 0$

3 Inventiones math., Vol. 14 
If $\Delta \subset N$ is a holomorphic disc with coordinate $z=r e^{i \theta}$, then (iii) is equivalent to the sub-mean-value property,

$$
\psi(0) \leqq \frac{1}{2 \pi} \int_{0}^{2 \pi} \psi\left(r e^{i \theta}\right) d \theta
$$

(2.9) Proposition. If $\psi$ is p.s.h. on $B_{n}^{*}$ and $n \geqq 2$, then $\psi$ extends to a p.s.h. function $\psi$ on the whole ball $B_{n}$.

Again, this proposition follows from general results about p.s. h. functions [9]. Here is a proof in our special case for $n=2$. Since $\psi \in L_{\mathrm{loc}}^{1}\left(B_{2}^{*}\right)$, we may define

$$
\psi(0)=\limsup _{\substack{\|z\| \| 0 \\ z \neq 0}} \psi(z)
$$

and we must show that $\psi(0)<+\infty$. From $(2.8)$, if $z_{1} \neq 0$ we have the estimate

$$
\psi\left(z_{1}, z_{2}\right) \leqq \frac{1}{2 \pi} \int_{0}^{2 \pi} \psi\left(z_{1}, z_{2}+\varepsilon e^{i \theta}\right) d \theta
$$

since the disc in question does not pass thru $z=0$. From this it follows easily that $\psi(0)<+\infty$. Q.E. D.

Finally, we shall need Fatou's lemma, which for our purposes may be stated as follows:

(2.10) Proposition. Let $\varphi=\frac{\sqrt{-1}}{2}\left(\sum_{j=1}^{n} d z_{j} \wedge d \bar{z}_{j}\right)$ be the Euclidean Kähler form on $\mathbf{C}^{n}$ and $\Phi=\frac{\varphi^{n}}{n !}$ the volume form. Let $\left\{\mu_{l}\right\}$ be a sequence of continuous functions on the (closed) ball $B_{n}$ which satisfy (i) $\mu_{l} \geqq 0$, (ii) the limit $\lim _{l \rightarrow \infty} \mu_{l}(z)=\mu(z)$ exists almost everywhere, and (iii) $\int_{B_{n}} \mu_{l} \Phi \leqq C<\infty$. Then $\mu \in L^{1}\left(B_{n}\right)$ and $0 \leqq \int_{B_{n}} \mu \cdot \Phi \leqq C$.

\section{Proof of Theorem I}

Let $f: B_{n}^{*} \rightarrow M$ be a holomorphic mapping into a compact Kähler manifold, and assume that $n \geqq 3$. Denote by $\omega$ the $(1,1)$ form on $M$ which is associated to the Kähler metric, and set $\omega_{f}=f^{*}(\omega)$. Then $\omega_{f}$ is a $C^{\infty}$ real $(1,1)$ form on $B_{n}^{*}$ and we have

$$
\begin{array}{r}
d \omega_{f}=0 \\
\omega_{f} \geqq 0 .
\end{array}
$$


Since the de Rham cohomology group $H^{2}\left(B_{n}^{*}, \mathbf{R}\right)=0$, we may write

$$
\omega=d \gamma
$$

where $\gamma$ is a real $C^{\infty} 1$-form on $B_{n}^{*}$.

Decomposing $\gamma=\gamma_{1,0}+\gamma_{0,1}$ into type, we have from (3.1) the relations

$$
\begin{aligned}
\gamma_{1,0} & =\bar{\gamma}_{0,1} \\
\bar{\partial} \gamma_{0,1} & =0=\partial \gamma_{1,0} \\
\omega_{f} & =\bar{\partial} \gamma_{1,0}+\partial \gamma_{0,1} .
\end{aligned}
$$

By Proposition (2.6) we may find a $C^{\infty}$ function $\eta$ on $B_{n}^{*}$ such that

$$
\bar{\partial} \eta=\gamma_{0,1}
$$

(this is where we use the assumption $n \neq 2$ ). If we now define

$$
\psi=\frac{\eta-\bar{\eta}}{2 \sqrt{-1}},
$$

then it follows from (3.2) and (3.3) that

$$
d d^{\mathfrak{c}} \psi=\omega_{f}
$$

where $d^{c}=\sqrt{-1}(\bar{\partial}-\partial)$. It follows that $\psi$ is a p.s.h. function on $B_{n}^{*}$, and by Proposition (2.9) $\psi$ extends to a p.s.h. function on $B_{n}$ (this is where we use the assumption $n \geqq 2$ ).

According to Proposition (2.4), we want to derive the estimates

$$
\int_{B_{n}^{*}}\left(d d^{c} \psi\right)^{k} \wedge \varphi^{n-k}=\int_{B_{n}^{*}}\left(\omega_{f}\right)^{k} \wedge \varphi^{n-k}<\infty \quad(k=1, \ldots, n) .
$$

Now even though $\psi$ extends across $z=0$ as a p.s.h. function, it may happen that

$$
\psi(0)=-\infty
$$

In fact, (3.6) exactly reflects the fact that the mapping $f: B_{n}^{*} \rightarrow M$ may be meromorphic and not holomorphic at $z=0$ (cf. proposition (3.10) below). For example, if we consider the residual map

$$
f: \mathbf{C}^{\mathbf{n}}-\{0\} \rightarrow \mathbf{P}_{n-1}
$$

then $\omega_{f}=d d^{c} \log \|z\|$ so that $\psi=\log \|z\|$ has a singularity at $z=0$. To get around this difficulty, I shall use a smoothing argument which was shown to me by Eli Stein. 
We choose a sequence of $C^{\infty}$ functions $\rho_{l}(z)$ which satisfy the conditions:

(i) $\rho_{l}(z) \geqq 0$ and $\int_{\mathbf{c}^{n}} \rho_{l}(z) \Phi(z)=1$.

(ii) Support $\left(\rho_{l}\right) \subset\left\{z \in \mathbf{C}^{n}:\|z\|<\frac{1}{l}\right\}$.

Recall now that $\psi$ is $C^{\infty}$ in the open punctured ball $\{z: 0<\|z\|<1+\varepsilon\}$ for some $\varepsilon>0$. Choose $l>\frac{1}{2 \varepsilon}$ and regularize $\psi$ by defining, for $z \in B_{n}$,

$$
\psi_{l}(z)=\int \psi(w) \rho_{l}(w-z) \Phi(w)
$$

or equivalently

$$
\psi_{l}(z)=\int \psi(w+z) \rho_{l}(w) \Phi(w) .
$$

These integrals make sense because a p.s.h. function is locally $L^{1}$. Eq. (3.7) shows that $\psi_{l}(z)$ is $C^{\infty}$ on $B_{n}$, and (3.8) shows that $\psi_{l}(z)$ is p.s.h. there. Furthermore, from (3.8) we have

$$
\lim _{l \rightarrow \infty} d d^{c} \psi_{l}(z)=d d^{c} \psi(z) \quad\left(z \in B_{n}^{*}\right),
$$

since $\psi$ is $C^{\infty}$ on $B_{n}^{*}$. Finally we have

$$
0 \leqq \int_{B_{n}}\left(d d^{c} \psi_{l}\right)^{k} \wedge \varphi^{n-k}=\int_{\partial B_{n}} d^{c} \psi_{l} \wedge\left(d d^{c} \psi_{l}\right)^{k-1} \wedge \varphi^{n-k} \leqq C<\infty
$$

because of (i) $d d^{c} \psi_{l} \geqq 0$, (ii) Stokes' theorem and $d \varphi=0$, and (iii) the fact that $\psi$ is $C^{\infty}$ near $\partial B_{n}$. The estimate (3.5) now follows from (3.9) and Proposition (2.10). Q.E.D.

Remark. To see how singular the p.s.h. function $\psi$ may be, we will prove the

(3.10) Proposition. Let $f: B_{n}^{*} \rightarrow M$ be a holomorphic mapping into a projective algebraic manifold $M$. Let $\omega$ be an arbitrary Kähler metric on $M$ and, assuming $n \geqq 3$, we write $\omega_{f}=f^{*}(\omega)$ and

$$
d d^{c} \psi=\omega_{f}
$$

for a p.s.h. function $\psi$ on $B_{n}$. Then we have

$$
|\psi(z)|=O\left(\log \frac{1}{\|z\|}\right) .
$$

Proof. Observe first that the estimate (3.12) is independent of the p.s.h. function $\psi$ which is a solution of (3.11). This follows from the fact that a real $C^{\infty}$ function $\lambda$ on $B_{n}^{*}$ which satisfies the equation

$$
d d^{c} \lambda=0
$$


is the real part of a holomorphic function $h(z)$ defined in $B_{n}^{*}$. Since $h(z)$ extends holomorphically across $z=0$ (Cauchy integral formula), the estimate (3.12) clearly depends only on $\omega_{f}$.

Now choose a Kähler metric $\omega^{\prime}$ on $M$ which is induced from a projective embedding $M \subset \mathbf{P}_{N}$. It follows that, for this metric,

$$
\omega_{f}^{\prime}=d d^{c} \psi^{\prime}
$$

where $\psi^{\prime}=\log \left(\left|g_{0}(z)\right|^{2}+\cdots+\left|g_{N}(z)\right|^{2}\right)$, the $g_{\alpha}(z)$ being holomorphic on $B_{n}$ and having no common zeroes except possibly the origin $z=0$. The conclusion (3.12) for the metric $\omega^{\prime}$ now follows from the elementary inequality

$$
\left|g_{0}(z)\right|^{2}+\cdots+\left|g_{N}(z)\right|^{2} \geqq c\|z\|^{\alpha} \quad(\alpha>0) .
$$

For an arbitrary Kähler metric $\omega$ on $M$, we can find an $\omega^{\prime}$ induced from a projective embedding such that

$$
\omega^{\prime}-\omega \geqq 0 \text {. }
$$

It follows that $\omega_{f}^{\prime}-\omega_{f} \geqq 0$, which in turn gives

$$
d d^{c}\left(\psi^{\prime}-\psi\right) \geqq 0 .
$$

Thus the function $\psi^{\prime}-\psi$ is p.s.h. on $B_{n}$, and from the maximum principle we have

$$
\psi^{\prime}-\psi \leqq C \text {. }
$$

The estimate (3.12) for $\psi$ now follows from the corresponding estimate for $\psi^{\prime}$. Q.E.D.

Remark. At this point we can explain the difficulty in proving that a holomorphic mapping $f: N-S \rightarrow M$ extends meromorphically when $\operatorname{codim}(S) \geqq 2$. Localizing, we may assume that $N$ is a open neighborhood of the origin in $\mathbf{C}^{n}$ and $S$ is an analytic set defined in $\bar{N}$. Our proof, together with the result of Shiffman discussed below (2.6), may be used to show that $f^{*} \omega=d d^{c} \psi$ where $\psi$ is a p.s.h. function on $\bar{N}$ which is $C^{\infty}$ on $N-S$. We want to show that

$$
\int_{N-S}\left(d d^{c} \psi\right)^{k} \wedge \varphi^{n-k}<\infty \quad(k=0, \ldots, n) .
$$

The convolution argument gives this when $\partial N \cap S=\Phi$ (i.e. $S$ has dimension zero), but this proof breaks down otherwise. Writing $\varphi=d d^{c}\|z\|^{2}$, the extendibility of $f: N-S \rightarrow M$ would follow from the following

Problem 0 . Let $N$ be a neighborhood of the origin in $\mathbf{C}^{n}$ and $S \subset \bar{N}$ an analytic subvariety. Let $\alpha$ be a p.s. h. function on $\bar{N}$ such that $\alpha$ is $C^{\infty}$ on $N-S$. Then do we have $\int_{N-S}\left(d d^{c} \alpha\right)^{n}<\infty$ ? 


\section{Statement and Discussion of Theorem II}

Let $N$ and $M$ be connected complex manifolds and let $S \subset N$ be an analytic subvariety with $U$ a sufficiently small open neighborhood of $S$ in $N$. We consider a holomorphic mapping

$$
f: N-U \rightarrow M,
$$

and are interested in the question of when such an $f$ extends to a holomorphic mapping from all of $N$ into $M$. Again we are primarily concerned with the case where $\operatorname{codim}(S) \geqq 2$, and we shall say that the image manifold $M$ obeys Hartogs' phenomenon when every such $f$ extends holomorphically across $U$ (cf. [6], p. 226).

Here are a few simple examples to illustrate the question. To give these we first observe that the problem is local around a point $x \in S$, and so we may assume that $N$ is a polycylinder in $\mathbf{C}^{n}, S$ is an analytic subvariety of $N$ given by holomorphic equations $h_{1}(z)=\cdots=h_{l}(z)=0$, and $U$ is the neighborhood of $S$ given by $\sum_{\mu=1}^{l}\left|h_{\mu}(z)\right|^{2}<\varepsilon$ for $\varepsilon$ sufficiently small.

Example 1. If $M=\mathbf{C}$, and if codim $(S) \geqq 2$, then $f$ in (4.1) extends to a holomorphic function on $N$ by the usual version of Hartogs' theorem.

Example 2. If $M=\mathbf{P}_{1}$ and if $\operatorname{codim}(S) \geqq 2$, then $f$ extends as a meromorphic mapping from $N$ to $\mathbf{P}_{1}$. In fact, $f$ defines a meromorphic function in $N-U$, and with a little effort it may be proved that $f$ factorizes as the quotient $\mathrm{g} / h$ of holomorphic functions $\mathrm{g}$ and $h$ defined in $N-U$. By Example 1 applied to $g$ and $h$, it follows that $f$ extends as a meromorphic function to all of $N$.

Example 3. If $M$ is a domain in $\mathbf{C}^{m}$ and if $\operatorname{codim}(S) \geqq 2$, then $f$ in (4.7) extends to a holomorphic mapping

$$
f: N \rightarrow E(M)
$$

where $E(M)=\operatorname{Spec}(\mathcal{O}(M))$ is the envelope of holomorphy of $M$ (cf. [6]). This follows from the inclusion

$$
f^{*}: \mathcal{O}(M) \rightarrow \mathcal{O}(N)
$$

implied by Example 1 .

Example 4. If codim $(S) \geqq 2$ and if $M$ is a projective algebraic variety, then $f$ in (4.7) extends meromorphically to $N$ by Examples 1 and 2 .

Example 5. Finally, if $M$ is the Hopf manifold given by Example 4 of Section 1, then Hartogs' phenomenon fails for $M$, as is exemplified by the residual mapping

$$
f: \mathbf{C}^{m}-\{0\} \rightarrow M .
$$


Our theorem is in response to a problem of Chern [3]. In order to state the result, we need to first review some notions from Hermitian differential geometry ([4], pp. 416-422). Thus let $V$ be an arbitrary complex manifold and $\mathbf{E} \rightarrow V$ a holomorphic vector bundle. Associated to an Hermitian metric in the fibres of $\mathbf{E} \rightarrow V$ there is a canonical Hermitian connection with curvature $\Omega_{\mathbf{E}}$. If $\mathbf{e}_{1}, \ldots, \mathbf{e}_{\mathbf{r}}$ is a local unitary frame for $\mathbf{E} \rightarrow V$ and $v_{1}, \ldots, v_{n}$ are local holomorphic coordinates on $V$, then we have an expansion

$$
\Omega_{\mathbf{E}}=\sum_{\rho, \sigma, i, j} \Omega_{\rho \sigma i j} \mathbf{e}_{\rho} \otimes \mathbf{e}_{\sigma}^{*} \otimes d v_{i} \wedge d \bar{v}_{j} .
$$

Using this we may define the bi-quadratic curvature form

by the formula

$$
\Omega_{\mathbf{E}}(e, \xi) \quad(e \in \mathbf{E}, \xi \in \mathbf{T}(V))
$$

$$
\Omega_{\mathbf{E}}(e, \xi)=\sum_{\rho, \sigma, i, j} \Omega_{\rho \sigma i \bar{j}} e_{\rho} \bar{e}_{\sigma} \xi^{i} \bar{\xi}^{j} .
$$

This curvature form has the following geometric interpretation: Given $\rho>0$, we define $\mathbf{E}(\rho)$ to be the tubular neighborhood of radius $\rho$ around the zero-cross-section of $\mathbf{E} \rightarrow V$. Thus $\mathbf{E}(\rho)=\{e \in \mathbf{E}:\|e\|<\rho\}$ where the length $\|e\|$ is measured using the given metric in $\mathbf{E}$. Then the curvature form $\Omega_{\mathbf{E}}(e, \xi)$ essentially gives the E.E. Levi form of $\partial \mathbf{E}(\rho)$ at the point $e$ (cf., [4], p. 426).

Now we take $V=M$ to be the complex manifold in which we are interested and $\mathbf{E}=\mathbf{T}(M)$ the holomorphic tangent bundle of $M$. The curvature form associated to an Hermitian metric $d s_{M}^{2}$ is then

$$
\Omega_{M}(\xi, \eta) \quad(\xi, \eta \in \mathbf{T}(M)) .
$$

For a $(1,0)$-tangent vector $\xi \in \mathbf{T}(M)$, the holomorphic sectional curvature $\Omega_{M}(\xi)$ in the 2-plane $\xi \wedge \bar{\xi}$ is given by (cf. [16])

$$
\Omega_{M}(\xi)=\Omega_{M}(\xi, \xi) .
$$

Definition. We shall say that $d s_{M}^{2}$ is negatively curved if all holomorphic sectional curvatures are non-positive (i. e., $\Omega_{M}(\xi) \leqq 0$ for all $\xi \in \mathbf{T}(M)$ ). Moreover, we will say that $d s_{M}^{2}$ is strongly negatively curved if the curvature form $\Omega_{M}(\xi, \eta) \leqq 0$ for all $\xi, \eta \in \mathbf{T}(M)$.

Obviously, if $d s_{M}^{2}$ is strongly negatively curved, then it is negatively curved but not conversely.

Theorem II. Suppose that $M$ is a complex manifold having a $d s_{M}^{2}$ which is complete and negatively curved. Then Hartogs' phenomenon is valid for $M$. 
Remark. This result has recently also been proved independently by Shiffman.

(4.4) Corollary. Let $M$ have a complete, negatively curved $d s_{M}^{2}$. Then any meromorphic mapping $f: N \rightarrow M$ is actually holomorphic.

This corollary follows from Theorem II by letting $S$ be the indeterminacy set of $f$.

(4.5) Corollary. Let $M$ have a complete, negatively curved $d s_{M}^{2}$ and let $N$ be a complete, rational algebraic variety. Then any holomorphic mapping $f: N \rightarrow M$ is constant.

Proof. Any such $N$ is bi-rationally equivalent to the projective space $\mathbf{P}_{n}$, and by Corollary (4.4) we may assume that $N=\mathbf{P}_{n}$. In the diagram

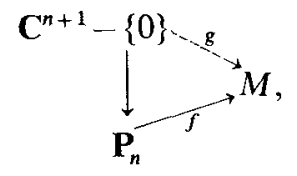

we may apply Theorem II to the holomorphic mapping $g$ and conclude that $f\left(\mathbf{P}_{n}\right)=g(0)$ is a point.

The proof of Theorem II will also give the

(4.6) Corollary. The Stein manifolds $M$ are exactly those complex manifolds such that (i) $\mathcal{O}(M)$ separates points and gives local coordinates, and (ii) $M$ carries a complete $d s_{M}^{2}$ with non-positive holomorphic sectional curvatures.

We will conclude this section with an example and a couple of open questions.

Example 6. Every Stein manifold $M$ carries a complete, negatively curved $d s_{M}^{2}$. In fact, we may use the embedding theorem [6] to realize $M$ as a closed submanifold of some $\mathrm{C}^{N}$. Then, the restriction to $M$ of the Euclidean $d s_{\mathbf{C}^{N}}^{2}$ has the desired properties (cf. Lemma (5.13) below). Thus our theorem covers the usual Hartogs' phenomenon given by Examples 1 and 5 above.

Problem 1. Is the Hartogs' phenomenon for meromorphic mappings true whenever $M$ is a compact Kähler manifold?

Referring to the proof of Theorem 1 given in Section 3 above, we may give a possible suggestion on how to show that a holomorphic mapping

$$
f: \partial B_{n}(\varepsilon) \rightarrow M \quad(n \geqq 3),
$$


with $M$ a compact Kähler manifold, extends meromorphically to the whole ball $B_{n}$. As in the proof of Theorem I we write

$$
d d^{c} \psi=\omega_{f}
$$

where $\omega_{f}=f^{*}(\omega)$ is the pull-back of the Kähler form on $M$ and $\psi$ is a $C^{\infty}$ p.s.h. function on $\partial B_{n}(\varepsilon)$. If we let

$$
U(\psi)=\left\{\eta: \eta \text { is p.s.h. on } B_{n} \text { and } \eta \leqq \psi \text { on } \partial B_{n}(\varepsilon)\right\},
$$

then the set $U(\psi)$ is non-empty, as may be seen by using the sub-meanvalue property $(2.8)$ for $\psi$. If we then let

$$
\Psi=\sup _{\eta \in U(\psi)}(\eta),
$$

it seems fairly plausible to me that $\Psi$ is a p.s.h. extension of $\psi$ to $B_{n}$. Assuming this, the regularizing argument of Section 3 would then show that the graph

$$
\Gamma_{f} \subset \partial B_{n}(\varepsilon) \times M
$$

of $f$ has finite volume relative to the metric $d s_{\mathbf{C}^{n}}^{2} \times d s_{\mathcal{M}}^{2}$. Thus, in order to carry out this proposed proof, we need to know the answer to

Problem 2. Let $V$ be an open submanifold in a complex manifold $W$. Assume that the boundary $\partial V$ is smooth and that the Levi form for $\partial V$ is $\leqq 0$ and has everywhere at least one negative eigenvalue. (Briefly, $\partial V$ is pseudo-concave.) Let $Z \subset V$ be a pure $k$-dimensional analytic set such that $\operatorname{vol}_{2 k}(Z)<\infty$, where this volume is computed with respect to a metric on $W$. Then does $Z$ locally extend across $\partial V$ ?

Remark. The extension of analytic sets across boundaries with pseudoconcavity assumptions has been discussed by Rothstein (Math. Ann. 133, 271-280 and 400-409 (1957)). It does not seem that his results contain the answer to Problem 2, although his techniques might be applicable.

\section{Proof of Theorem II}

We may write $S=S_{1} \cup \cdots \cup S_{K}$ as a disjoint union of complex submanifolds where $\operatorname{codim}\left(S_{\alpha}\right) \geqq \operatorname{codim}\left(S_{\alpha-1}\right)+1$ and where $S_{\alpha} \subset\left(S_{\alpha-1}\right)_{\text {sing }}$, the singular points of $S_{\alpha-1}$. (This is the usual stratification of an analytic variety.) Since the problem is local around a point $x \in S$, it will then suffice to assume that $S$ is smooth.

We now introduce the notations

$$
\begin{aligned}
\partial B_{k}(\varepsilon) & =\left\{z \in \mathbf{C}^{k}: 1-\varepsilon<\|z\| \leqq 1\right\}, \\
B_{l} & =\left\{w \in \mathbf{C}^{l}:\|w\| \leqq 1\right\} .
\end{aligned}
$$


Then locally around a point $x \in S, N-U$ is of the form $\partial B_{k}(\varepsilon) \times B_{l}{ }^{5}$ In order to isolate the essential aspects of the proof, we again take the extreme case $k=n$ and will thus give a proof of

Theorem II*. Let $f: \partial B_{n}(\varepsilon) \rightarrow M$ be a holomorphic mapping into a complex manifold $M$ which has a complete, negatively curved $d s_{M}^{2}$. Then, if $n \geqq 2, f$ extends to a holomorphic mapping from $\partial B_{n}\left(\varepsilon^{\prime}\right)$ into $M$ for some $\varepsilon^{\prime}>\varepsilon^{6}$

We will call $\partial B_{n}(\varepsilon)$ a spherical shell and we set

$$
S^{2 n-1}(\varepsilon)=\left\{z \in \mathbf{C}^{n}:\|z\|=1-\varepsilon\right\} .
$$

Then $S^{2 n-1}(\varepsilon)=\overline{\partial B_{n}(\varepsilon)}-\partial B_{n}(\varepsilon)$ is the inner boundary of $\partial B_{n}(\varepsilon)$. The proof of Theorem II* will now be given by a sequence of lemmas.

(5.1) Lemma. Suppose that $f$ extends continuously to

$$
\overline{\partial B_{n}(\varepsilon)}=\left\{z \in \mathbf{C}^{n}: 1-\varepsilon \leqq\|z\| \leqq 1\right\} .
$$

Then $f$ extends holomorphically to $\partial B_{n}\left(\varepsilon^{\prime}\right)$ for some $\varepsilon^{\prime}>\varepsilon$.

Proof. Let $z_{0} \in S^{2 n-1}(\varepsilon)=\overline{\partial B_{n}(\varepsilon)}-\partial B_{n}(\varepsilon)$ and set $w_{0}=f\left(z_{0}\right) \in M$. Take a polycylindrical coordinate neighborhood $P$ around $w_{0}$ in $M$, and assume that $P$ is given by $\left\{w \in \mathbf{C}^{m}:\left|w_{\alpha}\right|<1\right\}$. Then $f^{-1}(P) \cap \overline{\partial B_{n}(\varepsilon)}$ will contain a connected open neighborhood $\bar{U}$ of $z_{0}$ in $\partial B_{n}(\varepsilon)$ such that the restriction of $f$ to $U=\bar{U} \cap \partial B_{n}(\varepsilon)$ will be given by $m$ holomorphic functions $w_{\alpha} \circ f(\alpha=1, \ldots, m)$. By the usual argument utilizing the Cauchy integral formula (Kontinuitätssatz), each of these holomorphic functions may be extended to an open neighborhood $V$ of $z_{0}$ in $B_{n}$.

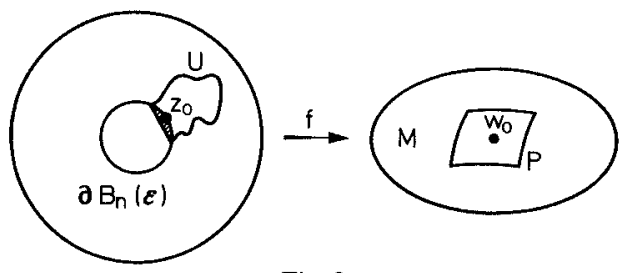

Fig. 2

${ }^{5}$ As before, a holomorphic mapping $f: \partial B_{k}(\varepsilon) \times B_{l} \rightarrow M$ is, by definition, given as a holomorphic mapping on the open set $\left\{(z, w) \in \mathbf{C}^{k} \times \mathbf{C}^{l}: 1-\varepsilon<\|z\|<1+\delta,\|w\|<1+\delta\right\}$ for some $\delta>0$. The obvious reason for this is that we are interested in the behavior of $f$ at the "inner boundary" of $\partial B_{n}(\varepsilon)$.

${ }^{6}$ This result may be compared with an (unpublished) theorem of $\mathrm{Wu}$, which states that if $M$ has a complete $d s_{M}^{2}$ with non-positive Riemannian sectional curvatures, then the universal covering manifold $M$ of $M$ is a Stein manifold (cf. [16]). For such an $M$, Hartogs' phenomenon is therefore true by Example 5 above. This result of Wu's will be discussed further in Appendix 2 below (cf. Proposition (A.2.17)). 
(In this picture $f$ extends across the boundary to the shaded region.) In other words, under the assumptions of the lemma, $f$ locally extends across the inner boundary $S^{2 n-1}(\varepsilon)$ of the spherical shell $\partial B_{n}(\varepsilon)$. Q.E. D.

The next three lemmas will lead to a proof of Theorem $1 I^{*}$ under the stronger assumption that $d s_{M}^{2}$ is complete and strongly negatively curved; i.e., that we have

$$
\Omega_{M}(\xi, \eta) \leqq 0
$$

for all $\xi, \eta \in \mathbf{T}(M)$. The function-theoretic meaning of this condition for holomorphic mappings is isolated in Lemma (5.9) below. Following this lemma, we shall return to the proof of Theorem II* in case $d s_{M}^{2}$ is complete and negatively curved. The function-theoretic meaning of this curvature assumption is given in Lemma (5.12).

(5.2) Lemma. Let $d s_{\mathbf{C}^{n}}^{2}$ be the usual flat metric on $\mathbf{C}^{n}$ and suppose that we have an estimate

$$
f^{*}\left(d s_{M}^{2}\right) \leqq C \cdot d s_{\mathbf{C}^{n}}^{2}
$$

Then $f$ extends continuously to $\overline{\partial B_{n}(\varepsilon)}$.

Proof. From (5.3) we have

$$
d_{M}\left(f(z), f\left(z^{\prime}\right)\right) \leqq C d_{\mathbf{C}^{n}}\left(z, z^{\prime}\right) \quad\left(z, z^{\prime} \in \partial B_{n}(\varepsilon)\right),
$$

where $d(.,$.$) denotes distance with respect to the particular metric in$ question. It follows from (5.4) that $f$ takes Cauchy sequences in $B_{n}$ into Cauchy sequences in $M$, and our lemma follows from the completeness of the metric on $M$. Q.E.D.

(5.5) Lemma. Let $\psi$ be a smooth function on $\partial B_{n}(\varepsilon)$ which satisfies $d d^{c} \psi \geqq 0$. Then $\psi \leqq C$ on all of $\partial B_{n}(\varepsilon)$, provided that $n \geqq 2$.

Proof. Such a function $\psi$ is pluri-sub-harmonic and satisfies the submean-value property (cf. (2.8))

$$
\psi(z) \leqq \int_{\xi \in \partial D(z)} \psi(\xi) d(\arg \xi)
$$

where $D(z)$ is a holomorphic disc with center $z$ and which lies entirely in $\partial B_{n}(\varepsilon)$. Since $n \geqq 2$, our lemma follows from (5.6). Q.E.D.

We now write

$$
f^{*}\left(d s_{M}^{2}\right)=\sum_{j, k} h_{j k} d z_{j} d \bar{z}_{k}
$$


where the Hermitian matrix $h=\left(h_{j k}\right)$ is $C^{\infty}$ in $\partial B_{n}(\varepsilon)$. Denote by $\lambda_{1} \leqq \cdots \leqq \lambda_{n}$ the (continuous) real eigenvalues of $h$ and let

$$
\sigma_{\mu}(f)=\sum_{i_{1} \leqq \cdots \leqq i_{\mu}} \lambda_{i_{1}} \ldots \lambda_{i_{\mu}}
$$

be the $\left(C^{\infty}\right) \mu^{\text {th }}$ elementary symmetric function of $\lambda_{1}, \ldots, \lambda_{n}$. Obviously we have

$$
f^{*}\left(d s_{M}^{2}\right) \leqq \sigma_{1}(f) d s_{\mathbf{C}^{n}}^{2},
$$

so that our theorem in the strongly negatively curved case will follow from Lemmas (5.2) and (5.5) together with the following

(5.9) Lemma. Assuming that $M$ is strongly negatively curved, the elementary symmetric functions $\sigma_{\mu}(f)$ satisfy $d d^{c} \sigma_{\mu}(f) \geqq 0$, and are therefore p.s.h. functions on $\partial B_{n}(\varepsilon)$.

Proof. This lemma follows from the formulae in Lu's paper [10].

Since we only need the result for $\sigma_{1}(f)$ (cf. (5.8)), we shall give the proof in this case. Let $\omega_{1}, \ldots, \omega_{m}$ be a $C^{\infty}$ local unitary co-frame on $M$ so that $d s_{M}^{2}=\sum_{\alpha=1} \omega_{\alpha} \bar{\omega}_{\alpha}$, and denote by $\Omega_{M}=\left\{\Omega_{\alpha \beta \gamma \delta}\right\}$ the curvature tensor on $M$ relative to this coframe. We set $f^{*}\left(\omega_{\alpha}\right)=\sum_{i} a_{\alpha i} d z_{i}$ so that $h_{i j}=$ $\sum_{\alpha} a_{\alpha i} \bar{a}_{\alpha j}$. From formula (4.19) in [10] we have

$$
\frac{\partial^{2} \sigma_{1}(f)}{\partial z_{j} d \bar{z}_{k}}=\sum_{\alpha, i} \frac{\partial a_{\alpha i}}{\partial z_{j}} \frac{\overline{\partial a_{\alpha i}}}{\partial z_{k}}+\sum_{\alpha, i} a_{\alpha i} b_{\alpha i j k},
$$

where, using Eq. (4.10) in [10],

$$
b_{\alpha i j k}=-\sum_{\beta, \gamma, \delta} \bar{a}_{\beta i} a_{\gamma j} \bar{a}_{\delta k} \Omega_{\alpha \beta \gamma \delta} .
$$

From (5.10) and (5.11) it follows that

$$
\begin{aligned}
\sum_{j, k} \frac{\partial^{2} \sigma_{1}(f)}{\partial z_{j} \partial \bar{z}_{k}} \xi_{j} \bar{\xi}_{k} & \geqq-\sum_{\substack{\alpha, \beta, \gamma, \delta \\
i, j, k}} a_{\alpha i} \bar{a}_{\beta i} a_{\gamma j} \bar{a}_{\delta k} \Omega_{\alpha \beta \gamma \delta} \xi_{j} \bar{\xi}_{k} \\
& =-\sum_{i}\left\{\sum_{\alpha, \beta, \gamma, \delta} \Omega_{\alpha \beta \gamma \delta} a_{\alpha i} \bar{a}_{\beta i}\left(\sum_{j} a_{\gamma j} \xi_{j}\right)\left(\overline{\sum_{k} a_{\delta k} \xi_{k}}\right)\right\} \\
& \geqq 0,
\end{aligned}
$$

where the last inequality follows from (4.2). Q.E.D. 
We now return to the proof of Theorem II* in case $d s_{M}^{2}$ is complete and negatively curved. As before, we will show that $f: \partial B_{n}(\varepsilon) \rightarrow M$ extends continuously to $\overline{\partial B_{n}(\varepsilon)}$ and then apply Lemma (5.1). The analogue of Lemma (5.9) which gives the geometric meaning of the holomorphic sectional curvature condition

is the following:

$$
\Omega_{M}(\xi) \leqq 0 \quad(\xi \in \mathbf{T}(M))
$$

(5.12) Lemma. Let $U$ be an open set in $\mathbf{C}^{n}$ and $f: U \rightarrow M$ a holomorphic mapping into a complex manifold having a negatively curved $d s_{M}^{2}$. Writing $f^{*}\left(d s_{M}^{2}\right)=\sum_{j, k=1}^{n} h_{j k} d z_{j} d \bar{z}_{k}\left(h_{j k}=\bar{h}_{k j}\right)$, we have $\frac{\partial^{2} h_{j j}}{\partial z_{j} \partial \bar{z}_{j}} \geqq 0$ on $U$.

Proof. Let $z \in U$ and let $D_{j}(z)$ be a holomorphic disc through $z$ given parametrically by $t \rightarrow\left(z_{1}, \ldots, z_{j}+t, \ldots, z_{n}\right)(|t|<\delta)$. If we set $f^{*}\left(d s_{M}^{2}\right) \mid D_{j}(z)$ $=h(t, \bar{t}) d t d \bar{t}$, then obviously

$$
\frac{\partial^{2} h_{j j}}{\partial z_{j} d \bar{z}_{j}}(z)=\frac{\partial^{2} h}{\partial t \partial \bar{t}}(0)
$$

so that it will suffice to prove the lemma in case $n=1$. Writing $f^{*}\left(d s_{M}^{2}\right)=$ $h d t d \bar{t}$ where $t$ is a coordinate on $U \subset \mathbf{C}$, we may assume that $f: U \rightarrow M$ is non-constant, and therefore $h$ vanishes only at isolated points of $U$. Obviously, it will suffice to prove the stronger statement that

$$
\frac{\partial^{2} \log h(t)}{\partial t \bar{\partial} t} \geqq 0
$$

at points $t$ where $h(t) \neq 0$. Localizing around such a point, we may assume that $f: U \rightarrow M$ is an embedding with image $S=f(U)$ a complex submanifold of $M$. Then $f^{*}\left(d s_{M}^{2}\right)=d s_{S}^{2}=d s_{M}^{2} \mid S$ is an Hermitian metric on the disc $S$ such that, by definition of $\Omega_{S}$,

$$
\frac{1}{h} \frac{\partial^{2} \log h}{\partial t \partial \bar{t}}=-\Omega_{S}\left(\frac{\partial}{\partial t}\right)
$$

i. e., $\frac{\partial^{2} \log h}{\partial t \partial \hat{t}}$ is minus the holomorphic sectional curvature of $S$ in the 2-plane $\frac{\partial}{\partial t} \wedge \frac{\bar{\partial}}{\partial t}$. Our proposition now follows from the

(5.13) Lemma ([4], p. 425). Let $M$ be a complex manifold with Hermitian metric $d s_{M}^{2}$, and let $S \subset M$ be a complex submanifold with induced 
metric $d s_{s}^{2}$. Then we have the relations

$$
\begin{aligned}
\Omega_{S}(\xi, \eta) & =\Omega_{M}(\xi, \eta)-|A(\xi \otimes \eta)|^{2} & & (\xi, \eta \in \mathbf{T}(S)), \\
\Omega_{S}(\xi) & =\Omega_{M}(\xi)-|A(\xi \otimes \xi)|^{2} & & (\xi \in \mathbf{T}(S))
\end{aligned}
$$

where $A$ is the $2^{\text {nd }}$ fundamental form of $S$ in $M$.

Remark. This lemma expresses a fundamental principal in Hermitian differential geometry to the effect that curvatures decrease on complex sub-manifolds.

We want to use Lemma (5.12) to show that $f: \partial B_{n}(\varepsilon) \rightarrow M$ extends continuously to $\overline{\partial B_{n}(\varepsilon)}$. Writing

$$
f^{*}\left(d s_{M}^{2}\right)=\sum_{j, k=1}^{n} h_{j k} d z_{j} d \bar{z}_{k},
$$

we first observe the elementary inequality

$$
f^{*}\left(d s_{M}^{2}\right) \leqq \sum_{j=1}^{n} h_{j j} d z_{j} d \bar{z}_{j}
$$

Denote by $\partial B_{n}(\varepsilon, \varepsilon / 2)$ the concentric spherical shell

$$
\left\{z \in \mathbf{C}^{n}: 1-\varepsilon<\|z\| \leqq 1-\varepsilon / 2\right\} .
$$

Let $z_{0} \in S^{2 n-1}(\varepsilon)$. By a unitary change of coordinates in $\mathbf{C}^{n}$, we may assume that $z_{0}=(1-\varepsilon, 0, \ldots, 0)$. Then the holomorphic tangent space $\mathbf{T}_{z_{0}}\left(S^{2 n-1}(\varepsilon)\right)$ to $S^{2 n-1}(\varepsilon)$ at $z_{0}$ is the $\mathbf{C}^{n-1}$ given parametrically by

$$
\left(v_{1}, \ldots, v_{n-1}\right) \rightarrow\left(1-\varepsilon, v_{1}, \ldots, v_{n-1}\right) .
$$

Thus the intersection $T_{z_{0}}\left(S^{2 n-1}(\varepsilon)\right) \cap \partial B_{n}(\varepsilon, \varepsilon / 2)$ is the punctured ball $B^{*}\left(z_{0}\right)$ given by $0<\left|v_{1}\right|^{2}+\cdots+\left|v_{n-1}\right|^{2} \leqq \varepsilon\left(1-\frac{3 \varepsilon}{4}\right)$.

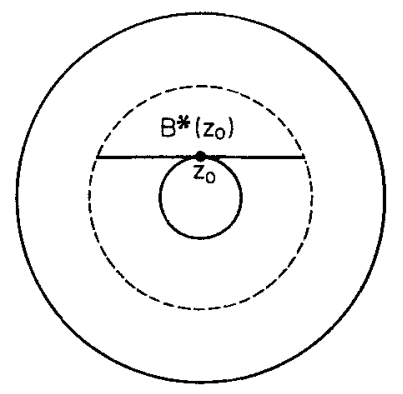

Fig. 3 
(5.15) Lemma. On $B^{*}\left(z_{0}\right)$ we have the estimate

$$
f^{*}\left(d s_{M}^{2}\right) B^{*}\left(z_{0}\right) \leqq c\left(d s_{\mathbf{C}^{n}}^{2}\right) \mid B^{*}\left(z_{0}\right)
$$

where the constant $c$ is independent of $z_{0}$.

Proof. In order to isolate the essential point, we shall consider the case $n=2$. From (5.14) we have the inequality

$$
f^{*}\left(d s_{M}^{2}\right) \mid B^{*}\left(z_{0}\right) \leqq h_{22} d z_{2} d \bar{z}_{2}
$$

since $d z_{1}=0$ on $B^{*}\left(z_{0}\right)$. Let $D\left(z_{0}, \delta\right)$ be the holomorphic disc in $\partial B_{2}(\varepsilon, \varepsilon / 2)$ given parametrically by $t \rightarrow(1-\varepsilon+\delta, t)$. On $D\left(z_{0}, \delta\right)$ we have $\frac{\partial^{2} h_{22}}{\partial t \partial \bar{t}} \geqq 0$

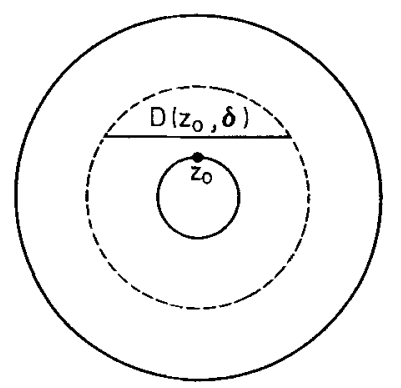

Fig. 4

by Lemma (5.12). It follows from the sub-mean-value property of subharmonic functions that

$$
h_{22}(1-\varepsilon+\delta, t) \leqq \frac{1}{2 \pi i} \int_{|\xi|=\varepsilon / 2} h_{22}(1-\varepsilon+\delta, t+\xi) \frac{d \xi}{\xi} .
$$

Letting $\delta \rightarrow 0$ in (5.16) we obtain the desired estimate. Q.E.D.

From Lemma (5.15) we obtain

$$
d_{M}\left(f(z), f\left(z^{\prime}\right)\right) \leqq d_{\mathbf{C}^{n}}\left(z, z^{\prime}\right) \quad\left(z, z^{\prime} \in B^{*}\left(z_{0}\right)\right),
$$

where the constant $c$ is independent of $z_{0} \in S^{2 n-1}(\varepsilon)$. It follows from (5.17) and the completeness of $d s_{M}^{2}$ that there is a point $w_{0} \in M$ such that, if $\left\{z_{\mu}\right\}$ is any sequence of points in $B^{*}\left(z_{0}\right)$ with $\lim _{\mu \rightarrow \infty} z_{\mu}=z_{0}$, then $\lim _{\mu \rightarrow \infty} f\left(z_{\mu}\right)=$ $w_{0}$. We then define $f\left(z_{0}\right)=w_{0}$, and it remains to show that this extended mapping $f: \overline{\partial B_{n}(\varepsilon)} \rightarrow M$ is continuous. This follows from our final

(5.18) Lemma. Let $\left\{z_{\mu}\right\}$ be any sequence of points in $\partial B_{n}(\varepsilon)$ with $\lim _{\mu \rightarrow \infty} z_{\mu}=z_{0}$. Then $\lim _{\mu \rightarrow \infty} f\left(z_{\mu}\right)=f\left(z_{0}\right)$. 
Proof. Again we take the case $n=2$. For $z_{\mu}$ close to $z_{0}$, there will be a (unique) $z_{\mu}^{\prime} \in S^{2 n-1}(\varepsilon)$ such that $z_{\mu} \in B^{*}\left(z_{\mu}^{\prime}\right)$. Furthermore, $B^{*}\left(z_{\mu}^{\prime}\right)$ and $B^{*}\left(z_{0}\right)$ will meet in a (unique) point $z_{\mu}^{\prime \prime}$.

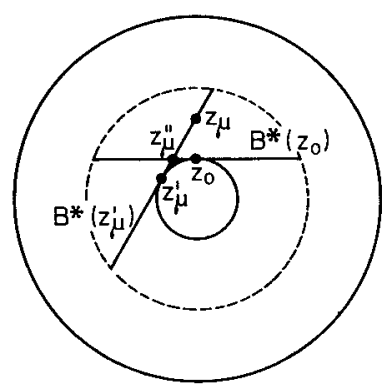

Fig. 5

By the triangle inequality on $M$,

$$
d_{M}\left(f\left(z_{\mu}\right), f\left(z_{0}\right)\right) \leqq d_{M}\left(f\left(z_{\mu}\right), f\left(z_{\mu}^{\prime \prime}\right)\right)+d_{M}\left(f\left(z_{\mu}^{\prime \prime}\right), f\left(z_{0}\right)\right) .
$$

Letting $\mu \rightarrow \infty$, both terms on the right-hand side of (5.19) tend to zero by (5.17). Q.E.D.

\section{Appendix I. Survey of Some Removable Singularity Theorems}

We want to discuss briefly the general problem of when a holomorphic mapping

$$
f: N-S \rightarrow M
$$

extends holomorphically or meromorphically across $S$. The case where $\operatorname{codim}(S) \geqq 2$ has been discussed in Sections 1 and 4 above.

The problem is local around a point $x \in S$. Utilizing Hironaka's resolution of singularities, we see that the essential case is when $N=$ $\left\{z=\left(z_{1}, \ldots, z_{n}\right) \in \mathbf{C}^{\boldsymbol{n}}:\left|z_{j}\right| \leqq 1\right\}$ is a polycylinder $P_{n}$ in $\mathbf{C}^{\boldsymbol{n}}$ and $S$ is the divisor $z_{1} \ldots z_{k}=0$. In this case $N-S$ is the punctured polycylinder

$$
P_{n}^{*} \cong\left(D^{*}\right)^{k} \times(D)^{n-k}
$$

where $D=\{z \in \mathbf{C}:|z| \leqq 1\}$ and $D^{*}=D-\{0\}$. Thus we will discuss the question of removable singularities for a holomorphic mapping

$$
f: P_{n}^{*} \rightarrow M \text {. }
$$

Example 1. The most classical case is the Riemann extension theorem [6], which says that $f$ in (A.1.1) extends to a holomorphic mapping $f$ : $P_{n} \rightarrow \bar{M}$ in case $M$ is a bounded open set in $\mathbf{C}^{n}$. (The question whether $f$ maps $P_{n}$ into $M$ instead of $\bar{M}$ is a question of the pseudo-convexity of $\partial M$.) 
Example 2. The mapping (A.1.1) extends holomorphically in case $M$ is compact and has a negatively curved $d s_{M}^{2}$. This basic result is due to Mrs. Kwack [8], whose proof is a variation on a previous argument of Grauert-Recksziegel. Another proof is given in Section 6 of [5]; this argument uses the Bishop-Stoll Theorem (2.2) above.

Observe also that Mrs. Kwack's theorem gives a proof of the usual Riemann extension theorem as follows: Replacing $M$ by a larger open set, we may assume that $M$ is a polycylinder in $\mathbf{C}^{m}$. Then there exists a properly discontinuous, fixed-point-free, group of holomorphic automorphisms $\Gamma$ acting on $M$ with compact quotient. By Mrs. Kwack's theorem, the map $f: P_{n}^{*} \rightarrow M / \Gamma$ extends holomorphically, and the result follows easily from this.

Example 3. In case $M=D / \Gamma$ is the quotient of a bounded, symmetric domain in $\mathbf{C}^{m}$ by an arithmetic group $\Gamma$, it is an unpublished result of Borel that the mapping (A.1.1) extends to a mapping from the closed polycylinder $P_{n}$ into the Borel-Baily compactification $\overline{D / \Gamma}$ of $D / \Gamma$ ([2]). This result includes the (big) Picard theorem as follows: Take $D=$ $\{z=x+i y: y>0\}$ to be the Poincaré upper-half-plane and $\Gamma=S L(2, \mathbf{Z})$ the modular group. Then (essentially) $D / \Gamma=\mathbf{P}_{1}-\{0,1, \infty\}$ and $\overline{D / \Gamma}=\mathbf{P}_{1}$, which gives the Picard theorem. The theorem of Borel has recently been generalized by Kobayashi-Ochiai [7].

Example 4. In case $M$ is an $n$-dimensional projective algebraic manifold with very ample canonical bundle, it was proved in [5] that a nondegenerate holomorphic mapping $f: P_{n}^{*} \rightarrow M$ extends to a meromorphic mapping from $P_{n}$ into $M$. (Note that this is the equi-dimensional case.) It was pointed out to me by Bombieri that essentially the same proof works if we only assume that the canonical bundle is ample. This latter result has been used by Carlson to give results on when a holomorphic mapping

$$
f: \mathbf{C}^{n} \rightarrow \mathbf{P}_{n}-H
$$

is degenerate, where $H$ is an algebraic hypersurface in $\mathbf{P}_{n}$. For example, if $\operatorname{deg}(H) \geqq n+3$ and $H$ is non-singular, then $f$ in (A.1.2) is degenerate. Moreover, this argument also works in case $H=L_{1} \cup \cdots \cup L_{n+3}$ is the union of $n+3$ linear hyperplanes in general position. In this case, Carlson almost obtains an affirmative answer to another one of Chern's problems [3], who asked if $f$ is degenerate in case $H$ is the union of $n+2$ linear hyperplanes in general position.

Example 5. In the case of a general holomorphic mapping (A.1.1) with $n \leqq \operatorname{dim}_{\mathbf{c}} M$, Carlson has proved results about removing singularities of non-degenerate mappings $f$ where assumptions are made on the $n^{\text {th }}$ exterior power $\Lambda^{n} \mathbf{T}^{*}(M)$ of the cotangent of $M$. Although not yet

4 Inventiones math., Vol. 14 
in final form, it seems likely that his methods will unify Examples 2 and 4 into one overall statement.

The recent book "Hyperbolic Manifolds and Holomorphic Mappings", Marcel Dekker, Inc. (1970), by Kobayashi also contains some discussion of removable singularity theorems which generalize the result of Kwack referred to above.

\section{Appendix II \\ Some Remarks on the Order of Growth of Holomorphic Mappings}

a) Formulation of the Problem

In general, a holomorphic mapping $f: N-S \rightarrow M$ certainly does not have a removable singularity along a sub-variety $S$ along which it is not defined, and it seems fairly clear that the most interesting aspect of holomorphic mappings involves studying the order of growth of $f$ along $S$, especially as this relates to the topological properties of $f$. In this appendix we shall discuss this problem and shall isolate what is to me the central open question, namely of finding the analogue of Bezout's theorem for several holomorphic functions.

Because this particular subject is not understood so well, it seems desirable to first consider perhaps the most important special case of the situation $f: N-S \rightarrow M$. Consequently, we will discuss a holomorphic mapping
(A.2.1)
$f: A \rightarrow M$

where $A$ is a smooth affine algebraic variety and $M$ is a smooth projective variety (thus $M$ is complete). Thus, e.g., we might have $A=\mathbf{C}^{n}$ and $M=\mathbf{P}_{m}$. In general, we may think of $A$ as being given in $\mathbf{C}^{N}$ by polynomial equations

$$
P_{\alpha}\left(z_{1}, \ldots, z_{N}\right)=0
$$

in such a manner that the projection

$$
A \stackrel{\pi}{\longrightarrow} \mathrm{C}^{n}
$$

realizes $A$ as an algebraic branched covering over $\mathbf{C}^{n}$.

Another way of viewing $A$ is by the smooth completions which it has. These are given by smooth, projective varieties $\bar{A}$ which contain $A$ as a Zariski open set such that $\bar{A}-A=D_{1} \cup \cdots \cup D_{K}$ is a union of smooth divisors with normal crossings:

$$
\begin{aligned}
& A \rightarrow \vec{A} \\
& \bar{A}-A=D_{1} \cup \cdots \cup D_{K} .
\end{aligned}
$$


Given two smooth completions $\bar{A}$ and $\bar{A}^{\prime}$, there is a third one $\bar{A}^{\prime \prime}$ and a commutative diagram of holomorphic mappings

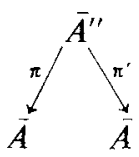

which are both the identity on $A$.

Then two methods (A.2.2) and (A.2.3) of viewing $A$ are both useful. Thus (A.2.2) allows us to see the global properties of $A$, such as the special pseudo-convex exhaustion function (cf., [15]).

$$
\tau(z)=\log \left(1+\left|z_{1}\right|^{2}+\cdots+\left|z_{N}\right|^{2}\right) ;
$$

while (A.2.3) allows us to localize at infinity. By the latter we mean that, letting $D=D_{1} \cup \cdots \cup D_{K}$ be the divisor at infinity on $A$, then a neighborhood in $A$ of a point $x \in D$ is a punctured polycylinder

$$
\begin{aligned}
& P_{n}^{*}=\left\{z=\left(z_{1}, \ldots, z_{n}\right):\left|z_{j}\right| \leqq 1, z_{1} \ldots z_{l} \neq 0\right\} \\
& P_{n}^{*} \cong\left(D^{*}\right)^{l} \times\left(D^{n-l}\right),
\end{aligned}
$$

which may be pictured for $n=l=2$ by

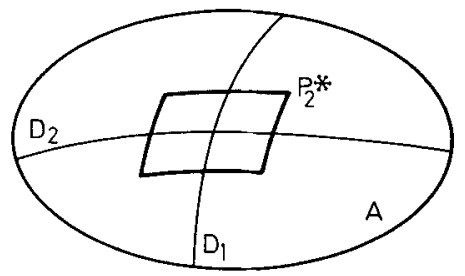

Fig. 6

If we restrict the exhaustion function $\tau$ to $P_{n}^{*}$, then we have

$$
\tau(z) \sim-\left(\log \left|z_{1}\right|+\cdots+\log \left|z_{l}\right|\right),
$$

where the notation " " means that each function is " 0 " of the other.

The diagram (A.2.4) is useful in proving that certain notions are independent of the smooth completion $\bar{A}$ of $A$. Thus, e.g., if we consider the mapping $\pi^{\prime \prime}: \bar{A}^{\prime \prime} \rightarrow \bar{A}$ in (A.2.4) localized at infinity, we have

$$
\pi^{\prime \prime}: P_{n}^{\prime *} \rightarrow P_{n}^{*}
$$

which is essentially given by equations

$$
z_{j}=\left(z_{1}^{\prime \prime}\right)^{\lambda_{j 1}} \ldots\left(z_{l^{\prime \prime}}^{\prime \prime}\right)^{\lambda_{j} l^{\prime \prime}} \quad(j=1, \ldots, l)
$$


where $D \cap P_{n}^{*}$ is given by $z_{1} \ldots z_{l}=0$ and $D^{\prime \prime} \cap P_{n}^{\prime *}$ is given by $z_{1}^{\prime \prime} \ldots z_{1}^{\prime \prime},=0$. It follows from (A.2.8) that $-\left(\log \left|z_{1}\right|+\cdots+\log \left|z_{l}\right|\right)$ is well-defined up to the relation " $\sim$ " explained above.

We want to study the amount of growth, or equivalently the amount of (essential) singularity at infinity, which a holomorphic mapping (A.2.1) has. This will be done relative to the following three auxiliary quantities: (i) the exhaustion function (A.2.5) and the associated level sets

$$
A[r]=\{z \in A: \tau(z) \leqq r\} ;
$$

(ii) a Kähler metric $d s_{M}^{2}$ on $M$ with $(1,1)$-form $\omega$ and pull-back $\omega_{f}=$ $f^{*}(\omega)$; and (iii) a Kähler metric $d s_{\bar{A}}^{2}$ on a smooth completion $\bar{A}$ of $A$ with $\varphi$ being the associated $(1,1)$ form.

\section{b) The Order Function for Holomorphic Mappings}

In a general manner, let $A$ and $M$ be complex manifolds of dimensions $n$ and $m$ respectively, and assume given: (i) an exhaustion function $\tau: A \rightarrow \mathbf{R}$ with Levi form $d d^{c} \tau$ and level sets $A[r]=\{z \in A: \tau(z) \leqq r\}$ (cf. [15]); (ii) an Hermitian metric $d s_{M}^{2}$ with $(1,1)$ form $\omega$; and (iii) an Hermitian metric $d s_{A}^{2}$ with $(1,1)$ form $\varphi$. Let $f: A \rightarrow M$ be a holomorphic mapping, $\omega_{f}=f^{*}(\omega)$, and introduce the quantities

and

$$
\begin{aligned}
v_{k}(f, r) & =\int_{A[r]} \omega_{f}^{k} \wedge \varphi^{n-k} \\
v\left(f ; r_{0}, \ldots, r_{n}\right) & =\sum_{k=0}^{n} v_{k}\left(f, r_{k}\right) \\
v(f, r) & =v(f ; r, \ldots, r),
\end{aligned}
$$

$$
\begin{aligned}
T_{k}(f, r) & =\int_{0}^{r} v_{k}(f, t) \frac{d t}{t} \\
T\left(f ; r_{0}, \ldots, r_{n}\right) & =\sum_{k=0}^{n} T_{k}\left(f, r_{k}\right) \\
T(f, r) & =T(f ; r, \ldots, r) .
\end{aligned}
$$

Definition. $T_{k}(f, r)$ is the $k^{\text {th }}$ order function for $f: A \rightarrow M$, and $T\left(f ; r_{0}, \ldots, r_{n}\right)$ is the total order function for this holomorphic mapping.

Referring to Proposition (2.5), we see that $v(f, r)$ is essentially the volume of that part $\Gamma_{f}[r]$ of the graph of $f$ which lies over $A[r]$, and where volume is computed relative to the product metric $d s_{A}^{2} \times d s_{M}^{2}$. The order functions $T_{k}(f, r)$ have been introduced because they appear naturally in the first main theorem (F.M.T.) to be discussed shortly. From the Bishop-Stoll Theorem (2.2) we have 
(A.2.11) Theorem. Let $A, M$ be algebraic varieties as discussed just above in Section (a). Then $f: A \rightarrow M$ is a rational map if, and only if,

$$
T(f, r)=O(\log r)^{7} .
$$

Example 1. In the simplest case where $A=\mathbf{C}$ and $M=\mathbf{P}_{1}, f$ is an entire meromorphic function and this theorem is given in Nevanlinna [12], p. 220.

There are two obvious questions regarding the order function $T\left(f, r_{0}, \ldots, r_{n}\right)$ : (i) How does $T$ depend on the choice of $\omega, \varphi$, and $\tau$ ? (ii) Which of the terms $T_{k}\left(f, r_{k}\right)$ is the more important? The answer to the first of these results from (A.2.4):

(A.2.12) Proposition. Different choices $\omega^{\prime}, \varphi^{\prime}, \tau^{\prime}$ lead to order functions $T_{k}^{\prime}(f, r)$ which satisfy

$$
\begin{aligned}
& T_{k}(f, r)=O\left(T_{k}^{\prime}\left(f, r^{\mu}\right)\right), \\
& T_{k}^{\prime}(f, r)=O\left(T_{k}\left(f, r^{v}\right)\right) .
\end{aligned}
$$

As to the second question, we shall give an example and then, following $\mathrm{Wu}$, a proposition to illustrate the converse to the example.

Example 2. Let $f: \mathbf{C}^{2} \rightarrow \mathbf{P}_{2}$ be the Fatou-Bieberbach mapping [1]. Then we have

$$
T_{2}(f, r)=O(\log r)
$$

since $f$ is one-to-one. On the other hand

$$
T_{1}(f, r) \neq O(\log r)
$$

since $f$ is not a rational mapping.

To give the proposition, we let $\varphi_{1}, \ldots, \varphi_{n}$ be a local unitary co-frame for $d s_{A}^{2}$ so that $d s_{A}^{2}=\sum_{j=1}^{n} \varphi_{j} \bar{\varphi}_{j}$. We then write

$$
f^{*}\left(d s_{M}^{2}\right)=\sum_{j=1}^{n} \lambda_{j} \varphi_{j} \bar{\varphi}_{j}
$$

where $0 \leqq \lambda_{1} \leqq \cdots \leqq \lambda_{n}$ are the (continuous) eigenvalues of $f^{*}\left(d s_{M}^{2}\right)$ with respect to $d s_{A}^{2}$. Letting $\sigma_{k}(f)=\sum_{i_{1} \leqq \cdots \leqq i_{k}} \lambda_{i_{1}} \ldots \lambda_{i_{k}}$ be the $k^{\text {th }}$ elementary symmetric function of the $\lambda_{j}$ s, we have

$$
\omega_{f}^{k} \wedge \varphi^{n-k}=\sigma_{k}(f) \cdot \varphi^{n}
$$

${ }^{7}$ In the case where $A$ is an affine algebraic variety as discussed above, we have $\int_{A} \varphi^{n}<\infty$ so that $T_{0}(f, r)=O(\log r)$. Referring to $(A .2 .5)$, we may in fact take $\varphi=d d^{c} t$ to be the Levi-form of $t$. 
which yields the relations

$$
\begin{aligned}
& v_{k}(f, r)=\int_{A[r]} \sigma_{k}(f) \cdot \Phi \\
& T_{k}(f, r)=\int_{0}^{r}\left\{\int_{A[t]} \sigma_{k}(f) \cdot \Phi\right\} \frac{d t}{t}
\end{aligned}
$$

where $\Phi=\varphi^{n}$ is the volume form on $A$.

Recall also Newton's inequalities

$$
\left(\sigma_{k}\right)^{1 / k} \leqq c_{k, l}\left(\sigma_{l}\right)^{1 / l} \quad(k \geqq l) .
$$

Definition (Wu). The holomorphic mapping $f: A \rightarrow M$ is said to be balanced if we have

$$
\left[v_{k}(r)\right]^{1 / k}=O\left(\left[v_{l}(r)\right]^{1 / l}\right) \quad(k \leqq l) .
$$

Note that (A.2.15) is very roughly the converse of the universal inequality (A.2.14). To explain more geometrically what it means for $f$ to be balanced, we observe that (A.2.15) is valid if $f$ is quasi-conformal in the sense that

$$
\lambda_{n}=O\left(\lambda_{1}\right) \text {. }
$$

The following proposition is due to $\mathrm{Wu}$. To state it, we let

$$
n(x, r)=\#\left\{f^{-1}(x) \cap A[r]\right\} \quad(x \in M)
$$

be the number of solutions of the equation $f(z)=x$ for $z \in A[r], x \in M$.

(A.2.16) Proposition. Let $f: A \rightarrow M$ be a balanced holomorphic mapping between algebraic varieties $A, M$ as in section a) above. (i)

$$
\left(\frac{T_{k}(f, r)}{\log r}\right)^{1 / k}=O\left(\frac{T_{l}(f, r)}{\log r}\right)^{1 / l} \quad(k \leqq l)
$$

(ii) if $\operatorname{dim}_{\mathbf{C}} A=\operatorname{dim}_{\mathbf{C}} M$, then $f(A)$ covers almost all of $M^{8}$, and (iii) if $n(r, x)=O(1)$ for all $x \in M$, then $f$ is rational.

Proof. Statement (i) follows from Hölder's inequality, and (ii) follows from [15]. As for (iii), we use (i) together with

$$
v_{n}(r)=\int_{x \in M} n(r, x) \omega^{n}(x)
$$

to find that $T_{k}(f, r)=O(\log r)$ for $k=1, \ldots, n$. The result now follows from Theorem (A.2.11). Q.E.D.

Roughly speaking, it seems that balanced holomorphic mappings should have the basic qualitative properties possessed by entire mero-

${ }^{8}$ This means that $M-f(A)$ has measure zero on $M$; i. e., the Casorati-Weierstrass property holds for $f: A \rightarrow M$. 
morphic functions. Some further indications of this will be given below (cf. Theorem (A.2.33)). Note that the Fatou-Bieberbach mapping is certainly not balanced, as follows from either (i) or (iii).

\section{c) The Maximum Modulus Function for Holomorphic Mappings}

The order function (A.2.10) measures the growth of a holomorphic mapping in terms of the area of the graph of $f$. Historically, this approach originated in Ahlfors-Shimizu interpretation of the Nevanlinna characteristic function of $f: \mathbf{C} \rightarrow \mathbf{P}_{1}$ in terms of the spherical image of $f$ (cf. [12], pp. 171-177). Long before this, it was customary to use the maximum modulus to measure the growth of an entire holomorphic function $f: \mathbf{C} \rightarrow \mathbf{C}$. We want to give a little generalization of this latter approach.

Thus, we let $M$ be a simply-connected complex manifold having a complete Hermitian metric which has non-positive Riemannian sectional curvatures $^{9}$. It follows from the theorem of Cartan-Hadamard that the geodesic balls

$$
M[\rho]=\left\{x \in M: d_{M}\left(x_{0}, x\right) \leqq \rho\right\}
$$

give an exhaustion of $M$ by convex regions with smooth boundaries. Moreover, it is a theorem of $\mathrm{Wu}$ (cf. the discussions in [16]) that the Levi form

$$
d d^{c} \log d_{M}\left(x_{0}, x\right) \geqq 0 \text {. }
$$

This leads to the following

(A.2.17) Proposition (Wu). Let $f: A \rightarrow M$ be a holomorphic mapping where $M$ is simply-connected and has a complete $d s_{M}^{2}$ with non-positive Riemannian sectional curvatures. Then the function

$$
\rho(f)(z)=\log d_{M}\left(f\left(z_{0}\right), f(z)\right)
$$

is pluri-sub-harmonic on $A$.

A similar proposition giving a geometric interpretation of the curvature forms $\Omega_{M}(\xi, \eta)$ and $\Omega_{A}(\xi, \eta)$ results from the computations in [10]. To give this we let $f: A \rightarrow M$ be a holomorphic mapping between complex manifolds having Hermitian metrics, and denote by $\sigma_{k}(f)$ the $k^{\text {th }}$ elementary symmetric function of the eigenvalues of $f^{*}\left(d s_{M}^{2}\right)$ with respect to $d s_{A}^{2}$.

(A.2.17)* Proposition. Assume that the curvature forms satisfy

$$
\begin{aligned}
& \Omega_{A}(\xi, \eta) \geqq 0, \\
& \Omega_{M}(\xi, \eta) \leqq 0 .
\end{aligned}
$$

Then the functions $\rho_{k}(f)=\log \sigma_{k}(f)$ are p.s.h. on $A$.

${ }^{9}$ From [16] we have that: $\{$ Riemannian sectional curvatures $\leqq 0\} \Rightarrow\{$ curvature form $\leqq 0\} \Rightarrow\{$ holomorphic sectional curvatures $\leqq 0\}$, and all implications are strict if $\operatorname{dim}_{c} M>1$. 
Proposition (A.2.17) suggests the

(A.2.18) Definition. Let $f: A \rightarrow M$ be a holomorphic mapping as in Proposition (A.2.17). Then the maximum modulus is defined by

$$
M(f, r)=\max _{z \in \hat{\partial} A[r]} \rho(f)(z)=\max _{z \in A[r]} \rho(f)(z) .
$$

Similarly, the mean value for $f: A \rightarrow M$ is

$$
m(f, r)=\int_{\partial A[r]} \rho(f) d^{c} \tau \wedge\left(d d^{c} \tau\right)^{n-1} .
$$

Remarks. The equality $\max _{z \in \partial A[r]} \rho(f)(z)=\max _{z \in A[r]} \rho(f)(z)$ follows from Proposition (A.2.17) and the maximum principle. In case we have $\Omega_{A}(\xi, \eta) \geqq 0$ and $\Omega_{M}(\xi, \eta) \leqq 0$, we may also define

$$
\begin{aligned}
M_{k}(f, r) & =\max _{z \in \partial A[r]} \rho_{k}(f)(z), \\
m_{k}(f, r) & =\int_{\partial A[r]} \rho_{k}(f) d^{c} \tau \wedge\left(d d^{c} \tau\right)^{n-1} .
\end{aligned}
$$

To give some properties of the maximum modulus and mean-value functions, we first introduce the

(A.2.19) Definition. The exhaustion function $\tau: A \rightarrow \mathbf{R} \cup\{-\infty\}$ is said to be a special exhaustion function if we have

$$
\begin{aligned}
d d^{c} \tau & \geqq 0, \\
\left(d d^{c} \tau\right)^{n} & =0 .
\end{aligned}
$$

Remark. To say that $\tau: A \rightarrow \mathbf{R} \cup\{-\infty\}$ is an exhaustion function means in particular that $A[r]=\{x \in A: \tau(x) \leqq r\}$ should be compact for every $r \in \mathbf{R}$. It is allowed that $\tau$ take on the value $-\infty$, just as is the case for p.s.h. functions.

Example 3. If $A=\mathbf{C}^{n}$, we may take $\tau(z)=\log \|z\|$ to have a special exhaustion function. More generally, if $A$ is any affine algebraic variety, then we may realize $A$ as a finite algebraic covering (cf. (A.2.2))

$$
\pi: A \rightarrow \mathbf{C}^{n},
$$

and may take $\tau(z)=\log \|\pi(z)\|$.

Remark. To some extent, the special exhaustion functions seem to be an analogue of the harmonic exhaustion functions which play such a crucial role in the theory of Riemann surfaces (cf. footnote ${ }^{14}$ below).

(A.2.20) Proposition. Let $A$ have a special exhaustion function $\tau$ and let $f: A \rightarrow M$ be a holomorphic mapping into a complex manifold $M$ as above. Then (i) $m(f, r)=O(M(f, r))$ and (ii) $m(f, r)$ is an increasing function of $r$. 
Proof. Observe first that, by $\left(d d^{c} \tau\right)^{n}=0$ and Stokes' theorem, the integral $\int_{1 \delta A[r]} d^{c} \tau \wedge\left(d d^{c} \tau\right)^{n-1}$ is independent of $r$. Also $d^{c} \tau \wedge\left(d d^{c} \tau\right)^{n-1} \geqq 0$ on $\partial A[r]$ since $d d^{c} \tau \geqq 0$. Thus we have

$$
m(f, r)=\int_{\partial A[r]} \rho(f) d^{c} \tau \wedge\left(d d^{c} \tau\right)^{n-1} \leqq M(f, r) \int_{\partial A[r]} d^{c} \tau \wedge\left(d d^{c} \tau\right)^{n-1},
$$

and (i) follows from this. To prove (ii), we have for $r_{2} \geqq r_{1}$

$$
\begin{aligned}
m\left(f, r_{2}\right)-m\left(f, r_{1}\right) & =\int_{\partial A\left[r_{2}\right]} \rho(f) d^{c} \tau \wedge\left(d d^{c} \tau\right)^{n-1}-\int_{\partial A\left[r_{1}\right]} \rho(f) d^{c} \tau \wedge\left(d d^{c} \tau\right)^{n-1} \\
& =\int_{A\left[r_{2}, r_{1}\right]} d \rho(f) \wedge d^{c} \tau \wedge\left(d d^{c} \tau\right)^{n-1} \\
& =\int_{A\left[r_{2}, r_{1}\right]} d \tau \wedge d^{c} \rho(f) \wedge\left(d d^{c} \tau\right)^{n-1} \\
& =\int_{r_{1}}^{r_{2}}\left\{\int_{\partial A[t]} d^{c} \rho(f) \wedge\left(d d^{c} \tau\right)^{n-1}\right\} d t \\
& =\int_{r_{1}}^{r_{2}}\left\{\int_{A[t]} d d^{c} \rho(f) \wedge\left(d d^{c} \tau\right)^{n-1}\right\} d t \\
& \geqq 0
\end{aligned}
$$

since $d d^{c} \rho(f) \geqq 0$. Q.E.D.

Remark. We should also have an estimate

$$
M(f, r)=O(m(f, k \cdot r)) \quad(k>1),
$$

but I don't know how to prove this except in special cases.

\section{d) Some Comments on the First Main Theorem}

Let $A$ be an affine algebraic variety as in a) above and denote by $\varphi$ the Kähler form coming from a smooth completion $\ddot{A}$ of $A$. If $V \subset A$ is a pure $k$-dimensional analytic sub-variety, then we define the order functions

$$
\begin{aligned}
& n_{V}(r)=\int_{V[r]} \varphi^{k} \\
& N_{V}(r)=\int_{0}^{r} n_{V}(t) \frac{d t}{t}^{10} .
\end{aligned}
$$

From the Bishop-Stoll Theorem (2.2) we have

10 The notations $n_{V}(r)$ and $N_{V}(r)$ are used to conform with traditional notations in value distribution theory [12]. 
(A.2.22) Proposition. $V$ is an algebraic sub-variety of $A$ if, and only if, $N_{V}(r)=O(\log r)$.

Let $f: A \rightarrow M$ be a holomorphic mapping of $A$ into a complex manifold $M$. In case $M$ is a compact Kähler manifold we have defined the order function $T\left(f ; \mathrm{r}_{1}, \ldots, r_{n}\right)$; and in case $M$ is simply-connected and has a complete $d s_{M}^{2}$ with non-positive Riemannian sectional curvatures, we have defined the maximum modulus $M(f, r)^{11}$. Both of these are notions measuring the order of growth of $f$, and both may be used to single out the rational maps in case $M$ is an algebraic variety. However, in order for these concepts to be fruitful, it is obviously necessary that they should lead to an interesting analysis of transcendental holomorphic mappings. This is certainly the case when $\operatorname{dim}_{\mathbf{c}} A=1$ [12], and is to some extent the case when $\operatorname{dim}_{\mathrm{c}} M=1$. However, it seems to me that, although there are several interesting results in the general case ([13] and [15]), the basic questions have yet to be grappled with successfully. I should like to briefly discuss what are, to me, these basic questions and then summarize briefly what seems to be known about them.

Thus let $M$ be a compact Kähler manifold (e.g. $\mathbf{P}_{m}$ ) and $f: A \rightarrow M$ a holomorphic mapping. Let $V \subset M$ be an algebraic sub-variety of codimension $q$ (e.g. $V=\mathbf{P}_{m-q}$ in case $M=\mathbf{P}_{m}$ ), set $V_{f}=f^{-1}(V)$. We assume that

$$
\operatorname{codim}_{x}\left(V_{f}\right)=q \quad\left(x \in V_{f}\right)^{12} .
$$

Problem A. Can we estimate $N_{V_{f}}(r)$ in terms of $T(f, r)$ ?

Example $3^{\prime}$. In case $A=\mathbf{C}$ and $M=\mathbf{P}_{1}$, this is the question of estimating the number of solutions of the equation

$$
f(z)=a
$$

in the disc $|z|<r$ and where $f(z)$ is an entire meromorphic function. Setting $N_{a_{f}}(r)=N(f, a, r)$, the first main theorem (F.M.T.) of Nevanlinna theory $[12]$ gives the estimate

$$
N(f, a, r)<T(f, r)+O(1) \quad\left(a \in \mathbf{P}_{1}\right),
$$

where the order function $T(f, r)$ is the integrated spherical image of $f$ and, in particular, is independent of the point $a \in \mathbf{P}_{1}$.

${ }^{11}$ Nothing essential will be lost from this discussion if we take $M=\mathbf{P}_{m}$ in the first case and $M=\mathbf{C}^{m}$ in the second.

12 This condition is equivalent to saying that $A \times V$ has proper intersection with the graph $\Gamma_{f}$ of $f$ in $A \times M$. We shall make this assumption throughout the following discussion. 
Example 4. In case $f: \mathbf{C} \rightarrow \mathrm{C}$ is an entire holomorphic function, then the maximum principle in the form of the Schwarz lemma gives the estimate

$$
n(f, o, r)<(\log 2) M(f, 2 r)
$$

on the number of zeroes of the holomorphic function $f(z)$.

Example 5. In case $A=\mathbf{C}$ and $M=\mathbf{P}_{m}$, we may let $V \subset \mathbf{P}_{m}$ be a linear hyperplane $\mathbf{P}_{m-1}$ and then there is a F.M.T. of the form (A.2.23) [13].

Example 6. In case $A$ is an arbitrary algebraic curve, then the statements of Examples 3, 4, 5 still remain valid, as may be seen by localizing in a punctured disc at infinity on $A$.

Example 7. In case $A$ is arbitrary algebraic variety and $M=\mathbf{P}_{1}$ or $M=\mathrm{C}$, then there are estimates of the form

$$
\begin{aligned}
& N(f, a, r)=O(T(f, r)) \quad\left(a \in \mathbf{P}_{1}\right) \\
& N(f, o, r)=O(M(f, r)) .
\end{aligned}
$$

These are obtained by localization in the punctured polycylinders at infinity of Jensen's formula in several complex variables.

Example 8. Finally, in case $A$ and $M$ are arbitrary algebraic varieties (with $M$ complete) and $V \subset M$ is a divisor, then we still have estimates similar to (A.2.25). Indeed, these may be seen to follow from Example 7.

In conclusion, from Examples 3-8 we may say that Problem $A$ is essentially $O . K$ in the case $V$ is of codimension one. (I do not mean to imply here that the really sharp quantitative results given by the second main theorem (S. M. T.) for $f: \mathbf{C} \rightarrow \mathbf{P}_{1}$ [12] have in any sense been pushed through in codimension one, but only that the qualitative information given by the classical F.M.T. holds in this case.) However, in the case where codim $(V)>1$, we do not seem to know the answer to Problem $A$. Even for the simplest cases

$$
\begin{array}{ll}
f: \mathbf{C}^{n} \rightarrow \mathbf{P}_{m} & (m>1), \text { or } \\
f: \mathbf{C}^{n} \rightarrow \mathbf{C}^{m} & (m>1) ; \\
V=\text { point, } &
\end{array}
$$

the answer to this problem seems mysteriously resistant. For instance, to be very concrete, let me state Bezout's problem for two holomorphic functions:

Problem $A^{\prime}$. Let $f(z, w)$ and $g(z, w)$ be two entire holomorphic functions of $(z, w) \in \mathbf{C}^{2}$ and assume that the divisors $f(z, w)=a$ and $g(z, w)=b$ have no common components. Then can we estimate the number of 
solutions of the equations

$$
\begin{aligned}
& f(z, w)=a \\
& g(z, w)=b \\
& |z|^{2}+|w|^{2}<r^{2}
\end{aligned}
$$

in terms of the growth of $f$ and $g$ ?

Example 9. In case $f$ and $g$ are polynomials of degrees $\alpha$ and $\beta$ respectively, then the number of common zeroes is $\leqq \alpha \cdot \beta$. This is the usual Bezout's theorem, and the reader may recall that the proof of this result (elimination theory) is considerably more difficult than the corresponding one-variable statement.

As positive evidence that Problem $\mathrm{A}^{\prime}$ should have some sort of answer, let me give the

(A.2.28) Proposition. Suppose that $f$ and $g$ are of finite exponential order and that $f: \mathbf{C}^{2} \rightarrow \mathbf{C}$ omits one value. Then Problem $A^{\prime}$ is $O . K$.

Proof. We shall only give the proof in case both $f$ and $g$ omit one value; the general argument is similar. By a linear change of coordinates, we may assume that $f$ and $g$ both omit the value 0 . Then we have

$$
\begin{aligned}
& f(z, w)=e^{2 \pi i P(z, w)} \\
& g(z, w)=e^{2 \pi i Q(2, w)}
\end{aligned}
$$

where $P, Q$ are polynomials whose degrees give the orders of $f, g$ respectively. Writing $a=e^{2 \pi i \alpha}, b=e^{2 \pi i \beta}$, the solutions to (A.2.27) are given by points $(z, w)$ which satisfy

$$
\begin{array}{ll}
P(z, w)=\alpha+k, & k \in \mathbf{Z} \\
Q(z, w)=\beta+l, & l \in \mathbf{Z} \\
|z|^{2}+|w|^{2}<r^{2} . &
\end{array}
$$

Using Example 7, it is easy to see that the number of solutions to (A.2.29) is $O\left(r^{2} \operatorname{deg} P \cdot \operatorname{deg} Q\right)$. Q. E. D.

There is a F.M.T. for a holomorphic mapping $f: \mathbf{C}^{n} \rightarrow \mathbf{P}_{m}$ and $V=\mathbf{P}_{m-q}$ which is due to Chern and $\mathrm{Wu}(n=m=q)$ and Stoll (any $n, m$, and $q$ ); cf. $[13,15]$ and the references given there ${ }^{13}$. In the present context, this result is given by the formula $\left(r_{0}<r\right)$

$$
\text { (A.2.30) } N_{V_{f}}(r)+m(f, V, r)=T_{q}(f, r)+m\left(f, V, r_{0}\right)+S(f, V, r)+O(1)
$$

${ }^{13}$ Both Wu's and Stoll's theorems are more general than the case being considered here. Especially Stoll's F.M.T. includes all known cases. It is necessary to include the multiplicity of $V_{f}$ in the counting function given in (A.2.30). 
where the counting function $N_{V_{f}}(r)$ is given by (A.2.21) with $k=n-q=$ $\operatorname{dim}_{\mathbf{C}} V_{f}$, the order function $T_{q}(f, r)$ is given by (A.2.10), and the remaining terms are given by

(A.2.31)

$$
\begin{aligned}
& m(f, V, r)=\int_{\mathbf{C}^{n}[r]} f^{*}(\Lambda(V)) \wedge d^{c} \tau \wedge \varphi^{n-q} \geqq 0 \\
& S(f, V, r)=\int_{\mathbf{C}^{n}[r]} f^{*}(\Lambda) \wedge d d^{c} \tau \wedge \varphi^{n-q} \geqq 0
\end{aligned}
$$

where $\mathbf{C}^{n}[r]=\left\{z \in \mathbf{C}^{n}:\|z\|<r\right\}, \tau(z)=\log \|z\|$, and where $A(V)$ is a certain $(q-1, q-1)$ form on $\mathbf{P}_{m}$ which has singularities along $V=\mathbf{P}_{m-q}{ }^{14}$. The F.M.T. (A.2.30) leads to the inequality

$$
\text { (A.2.32) } \quad N_{V_{f}}(r)<T_{q}(f, r)+S(f, V, r)+m\left(f, V, r_{0}\right)+O(1) \text {, }
$$

and it is probably reasonable to try and discount the effect of term $m\left(f, V, r_{0}\right)$ since $r_{0}$ is being held fixed. Even if this is done, we still don't know which of the terms $T_{q}(f, r)$ or $S(f, V, r)$ is the more important, and indeed the Fatou-Bieberbach example shows that the effect of the term $S(f, V, r)$ (which, contrary to $T_{q}(f, r)$, depends on the particular $V$ ) cannot be ignored. The best indication I know of their relative importance is the following

(A.2.33) Theorem (Chern-Stoll-Wu [13]), If we have

$$
\lim _{r \rightarrow \infty}\left[\frac{v_{q-1}(f, r)}{T_{q}(f, r)}\right]=0
$$

then the image $f\left(\mathbf{C}^{n}\right)$ intersects almost all linear subspaces $\mathbf{P}_{m-q}$ in $\mathbf{P}_{m}$. In particular, this is true if $f$ is balanced (cf. (A.2.15)).

Leaving aside Problem A for the moment, let me return again to the use of the order function $T\left(f ; r_{1}, \ldots, r_{n}\right)$ to measure the growth of $f: A \rightarrow M$ with $M$ a compact Kähler manifold.

Problem B. Does the order function $T(f)$ have good functorial properties? In particular, given two mappings $f_{1}: A \rightarrow M_{1}$ and $f_{2}: A \rightarrow M_{2}$, can we estimate $T\left(f_{1} \times f_{2}\right)$ for the product mapping $f_{1} \times f_{2}: A \rightarrow M_{1} \times M_{2}$ in terms of $T\left(f_{1}\right)$ and $T\left(f_{2}\right)$ ?

Remark. It is trivial to estimate the order function for

$$
f_{1} \times f_{2}: A \times A \rightarrow M_{1} \times M_{2}
$$

in terms of $T\left(f_{1}\right)$ and $T\left(f_{2}\right)$. Using the diagonal embedding $A \rightarrow A \times A$, we see that Problem $\mathrm{B}$ is implied by

Problem $B^{\prime}$. Let $f: A \rightarrow M$ be a homomorphic mapping into a compact Kähler manifold and let $B$ be an algebraic sub-variety of $A$. Then can we estimate $T(f \mid B)$ in terms of $T(f)$ ?

${ }^{14}$ In case $q=1$, we may replace $\varphi$ in (A.2.31) by $d d^{c} \log \|z\|$ and use $\left(d d^{c} \log \|z\|\right)^{n}=0$ to eliminate the term $S(f, V, r)$. This suggests why the case $\operatorname{codim}(V)=1$ should be $\mathrm{O} . \mathrm{K}$. 
Finally, to better understand Problems A, $\mathrm{A}^{\prime}$ and B, B', let me give one last problem which includes them all.

Problem $C$. Let $A$ be an algebraic variety and let $V, W$ be pure-dimensional analytic sub-varieties such that the intersection $V \cap W$ is defined. Then can we estimate the volume $\operatorname{vol}(V \cap W)$ in terms of $\operatorname{vol}(V)$ and $\operatorname{vol}(W)$ ?

Remarks. (i) By using the diagonal construction given above, we may assume that either $V$ or $W$ is an algebraic sub-variety. (ii) By localization at infinity, we see that Problem C (and therefore all of the other problems) are local questions in a punctured polycylinder. Further reductions of this sort show that the essential question is exactly the Bezout Problem A'.

\section{References}

1. Bochner, S., Martin, W.: Several complex variables. Princeton University Press 1948.

2. Baily, W., Borel, A.: Compactifications of arithmetic quotients of bounded symmetric domains. Ann. of Math. 84, 442-528 (1966).

3. Chern, S. S.: Differential geometry - its past and future, to appear in Proc. Nice Congress.

4. Griffiths, P.A.: The extension problem in complex analysis: II. Amer. J. Math. 88, $366-446$ (1966).

5. - Holomorphic mappings into canonical algebraic varieties, Ann of Math. 93, 439-458 (1971).

6. Gunning, R., Rossi, H.: Analytic functions of several complex variables. Englewood Cliffs, New Jersey: Prentice-Hall 1965.

7. Kobayashi, S., Ochiai, T.: Satake compactification and the great Picard theorem, to appear.

8. Kwack, M.: Generalization of the big Picard theorem. Ann. of Math. 90, 13-22 (1969).

9. Lelong, P.: Fonctions plurisousharmoniques et formes différentielles positives. New York: Gordon and Breach 1968.

10. Lu, Y.: Holomorphic mappings of complex manifolds. Jour. of Diff. Geom. 2, 299-312 (1968).

11. Narasimhan, R.: Introduction to the theory of analytic spaces, lecture notes No. 25. Berlin-Heidelberg-New York: Springer 1966.

12. Nevanlinna, R.: Analytic functions. Berlin-Heidelberg-New York: Springer 1970.

13. Stoll, W.: Value distribution of holomorphic maps. Several complex variables I, lecture notes No. 155, pp. 165-190. Berlin-Heidelberg-New York: Springer 1970.

14. Stolzenberg, G.: Volumes, limits, and extensions of analytic varieties, lecture notes No. 19. Berlin-Heidelberg-New York: Springer 1966.

15. Wu, H.: Remarks on the first main theorem of equidistribution theory I, II, IIL Jour. of Diff. Geom. 2, 197-202 (1968); 3, 83-94 (1969); and 3, 369-384 (1969).

16. Normal families of holomorphic mappings. Acta Math. 119, 193-233 (1967).

P.A. Griffiths

Princeton University

Department of Mathematics

Princeton, New Jersey 08540, USA 